\title{
THE FUNCTIONAL PROPERTIES OF THE LIGHT-SENSITIVE NEURONS OF THE POSTERIOR PARIETAL CORTEX STUDIED IN WAKING MONKEYS: FOVEAL SPARING AND OPPONENT VECTOR ORGANIZATION ${ }^{1}$
}

\author{
BRAD C. MOTTER ${ }^{2}$ AND VERNON B. MOUNTCASTLE
}

Department of Physiology, The Johns Hopkins University, Baltimore, Maryland 21205

\begin{abstract}
We describe in this paper the results of a new study of the inferior parietal lobule in 10 waking monkeys combining the methods of behavioral control, visual stimulation, and single neuron analysis. In this study, 1682 neurons were identified; 804 were studied in detail. Neurons insensitive to visual stimuli comprise the fixation, oculomotor, and projection-manipulation classes thought to be involved in initiatives toward action. The largest group of the light-sensitive (LS) neurons were activated from large and frequently bilateral response areas that excluded the foveal region; we term this foveal sparing. The remaining cells subtended areas including the fovea, when tested with large stimuli $\left(6^{\circ} \times 6^{\circ}\right)$, but only 8 of 216 cells studied in detail responded to the small fixation target light. We propose that a dynamic central neural process associated with the acts of fixation and visual attention suppresses responses to foveal stimuli.

Parietal LS neurons are sensitive to stimulus movement and direction over a wide range of velocities. The vectors point either inward toward the center or outward toward the perimeter of the visual field, and for neurons with bilateral response areas, the vectors commonly point in opposite directions in the two halffields; we term this opponent vector organization.

The functional properties of area $7 \mathrm{LS}$ neurons are such that they could signal motion in the immediate surround and the apparent motion accompanying head movements and forward locomotion. We surmise that they contribute to a central neural image of immediately surrounding space and to the perceptual constancy of that space obtaining during bodily movement. These properties are suitable for the attraction of gaze and attention to objects and events in the peripheral visual fields. It is this system, together with the classes of parietal neurons concerned with action initiatives, whose destruction is thought to account for the hemi-inattention and neglect of the parietal lobe syndrome in primates.
\end{abstract}

A number of studies have been made of the homotypical cortex of the inferior parietal lobule in waking monkeys. The animals used in these experiments were in most cases trained to emit stereotyped behavioral acts surmised on other grounds to be controlled or influenced by neural systems of which this cortical region is a part (Goldberg and Robinson, 1977, 1978; Hyvarinen and Poranen, 1974; Hyvarinen and Shelepin, 1979; Leinonen and Nyman, 1979; Leinonen et al., 1979; Lynch et al., 1973a, b, 1977; Mountcastle et al., 1975; Robinson and Goldberg, 1977a, b; Robinson et al., 1978; Sakata et al., 1977, 1978, 1980; Yin and Mountcastle, 1977). The results obtained have been considered in the light of changes in behavior that follow lesions of this region in man and in monkeys. They have led to several different but related concepts of the function of this region of the homotypical cortex.

\footnotetext{
' This work was supported by a grant from the United States Public Health Service (5 RO 1 EY03167) which we gratefully acknowledge. Correspondence and requests for reprints should be addressed to V. B. Mountcastle.

${ }^{2}$ Fellow of the United States Public Health Service, 1978-1980.
}

The first idea is that this region (area 7 in the rnonkey) functions as an association cortex, in the traditional sense, in which neural abstractions of sensory input signals converge and are "integrated." The resulting neural activity is then regarded as leading to, or itself to be, the neural basis of perceptual experiences. Neurons with convergent properties have been observed in area 7 , although cells of this type make up only a small percentage of all the cells identified (Hyvarinen and Poranen, 1974; Leinonen and Nyman, 1979; Leinonen et al., 1979; Lynch et al., 1973a, b, 1977; Mountcastle et al., 1975). Moreover, the studies made so far have shown only the fact of convergence, on which basis alone neurons in many parts of the neuraxis might equally well qualify for an "associative" function. It remains to be shown on quantitative grounds in what way the activities of these cells, evoked by a variety of stimuli, might provide some higher order or abstracted replicate of a complex sensory event, e.g., a pattern of activity uniquely defined by a certain spatial and temporal combination of stimuli in two different sensory domains. Convergence alone does 
not establish the case. Thus, while it is likely that the parietal homotypical cortex plays an important role in what are called associative functions, the neural mechanisms of those associations are still unclear.

The second general concept is that the inferior parietal lobule is a higher order processing area of the visual system, for it is known to receive convergent inputs from both the geniculostriate and the collicular portions of the visual system. The striate and the prestriate areas are believed to project upon the inferior parietal lobule over a multiple-stage, transcortical system. Area 7 also receives and processes neural signals transmitted via the retino-collicular system and its upward thalamocortical projections, signals thought to provide information about the spatial location of objects, not their contour, orientation, or color. This idea is supported by the fact that neurons activated by visual stimuli were observed in the earliest studies of the inferior parietal lobule (Hyvarinen and Poranen, 1974; Lynch et al., 1973a; Mountcastle et al., 1975) and have since been studied by Goldberg and Robinson (1977, 1978), Robinson and Goldberg (1977a, b), Robinson et al. (1978), and Yin and Mountcastle (1977). The light-sensitive neurons of area 7 make up 25 to $30 \%$ of the cells in the region whose functional properties can be identified in experiments of the sort described here.

The third idea is that additional functions of the inferior parietal lobule relate to more complex aspects of behavior and especially to the representation of and operations within immediately surrounding space, i.e., in spatial orientation and perception. For example, the region is thought to be involved in the combined actions of hand and eye within the immediately surrounding behavioral space and, more generally, with maintaining relations between internal bodily and external spatial coordinate systems. There is considerable evidence to suggest that this area, together with the cortical and subcortical structures with which it is linked, plays a role in the direction of attention and in the interested fixation of gaze usually but not always coincident with the direction of attention (Mountcastle, 1976, 1978). These ideas are based upon the behavioral deficits of primates with parietal lesions and the functional properties of several large classes of neurons, other than light-sensitive cells, that have been identified in area 7 (Hyvarinen and Poranen, 1974; Leinonen et al., 1979; Mountcastle et al., 1975; Lynch et al., 1973a, b, 1977; Sakata et al., 1977, 1978, 1980; Yin and Mountcastle, 1977).

The present study is an extension of those made earlier by ourselves and by others of the inferior parietal lobule. It was carried out under experimental conditions that allowed us to define more precisely the functional properties of the light-sensitive neurons of area 7 particularly in regard to the organization of their response areas and their sensitivity to movement and the direction of movement of luminous stimuli. We sought to determine from these properties the suitability of the system for controlling several components of visual behavior: for the attraction of visual attention, particularly for moving objects; for combined visual and manual operations; for spatial orientation and perception. The results obtained are described in the present paper; a short note has appeared (Motter and Mountcastle, 1979).

In the course of these experiments, we discovered a powerful effect of the act of fixation upon the excitability of the parietal light-sensitive neurons and, in addition, we found that their sensitivity to light stimuli is influenced by the angle of gaze, even though the large majority are related to retinotopic response areas (Andersen and Mountcastle, 1980; Motter and Mountcastle, 1980). Therefore, we have examined again the large class of parietal cells found by a number of investigators to be active during the interested fixation of gaze and insensitive to visual stimuli (Hyvarinen and Poranen, 1974; Lynch et al., 1973a, b; Mountcastle et al., 1975; Rolls et al., 1979; Sakata et al., 1977). We have confirmed those properties of the fixation neurons under a number of controlled conditions and compared them with the effects of fixation and the angle of gaze upon the light-sensitive neurons. These results will be described in a later paper.

\section{Methods}

The behavioral paradigms used in our previous experiments (Mountcastle et al., 1975; Lynch et al., 1977; Yin and Mountcastle, 1977) were designed with reference to the behavioral deficits produced in man and in monkeys by lesions of the posterior parietal cortex (for review, see Lynch, 1980). We added tests for the present experiments using a variety of visual stimuli that allowed us to study the visual responses of neurons of area 7 during the performance of visuomotor tasks.

Behavioral tasks and test equipment. Macaque monkeys were trained to detect the dimming of a small red target light. Behavioral trials were initiated with computer control, progressed when the monkey closed a response key, and were terminated by release of the key after the monkey detected the dimming of the target light. Constraints upon response times, together with variable foreperiods, eliminated timing as a cue. The monkeys were required to make appropriate eye movements to maintain fixation of the target light if it was displaced and not to break fixation when other visual stimuli appeared. A criterion of $90 \%$ correct performance on the visuomotor/detection tasks was reached by successive approximations. One to three weeks before the recording experiments began silver-silver chloride (electro-oculographic, EOG) electrodes were implanted in the orbital rims, and a head restraint device was fixed to the monkey's skull in a sterile operative procedure under anesthesia. Final training with the head fixed and with collection of eye movement records allowed us to set a final performance level. The use of near-threshold dimming detection levels, a wide variety of tasks, and randomly interspersed catch trials ensured continued correct performance of the tasks. For monkeys 84,86 and 87 , eye position was monitored and behavioral trials were terminated automatically through computer control if inappropriate eye movements occurred. In a second operative procedure, 2 to 3 days before recording began, a microelectrode recording chamber was placed over a bony opening centered on the inferior parietal lobule. When study of the first hemisphere was completed, a similar chamber placement was made on the second side, and the experiment continued.

The experimental apparatus for series A of Table I was described earlier (Lynch et al., 1977). It consisted of a tangent screen upon which a laser-generated fixation target could be projected and moved about by galvanom- 
eter mirrors. For other tests, the tangent screen could be replaced by a white opaque screen embedded with 17 light-emitting diodes (LEDs) arranged in various spatial patterns. The visual angle subtended by these displays could be varied from $36^{\circ}$ to $60^{\circ}$. In addition, a narrow, curved, white opaque board embedded with LEDs could be mounted $31 \mathrm{~cm}$ in front of the animal; it would extend $76^{\circ}$ into the peripheral visual field. The long axis of the board could be rotated through the visual field in $45^{\circ}$ steps.

A second test apparatus was developed for experiments 81 to 83 of series B, listed in Table I. It allowed the presentation of stationary or moving light stimuli of various shapes, sizes, and intensities at positions up to $90^{\circ}$ away from the central line of gaze. The monkey viewed binocularly one of three screens arranged like the adjacent sides of a regular hexagon, with the monkey at the center at a viewing distance of $54 \mathrm{~cm}$ from each screen center. Each $60^{\circ} \times 60^{\circ}$ screen surface consisted of a gray, patternless, back projection material, although only the two side screens were used as projection surfaces. When the monkey faced the center screen, the field of view was free laterally for $\pm 100^{\circ}$ and vertically for $\pm 30^{\circ}$. A $0.3^{\circ}$ fixation target was generated by a heliumneon laser $(\lambda=633 \mathrm{~nm}, 0.5 \log$ unit above background luminance). The laser spot could be directed under program control to any position on any of the three screens by deflection off mirror galvanometers and a rotatable turret located above the animal's head. The monkey could be turned to face either side screen to allow exploration of the visual field near the point of fixation.

For monkeys 84,86 , and 87 , the three-screen arrange-

TABLE I

The data base for the present experiments

Series A was carried out in the apparatus described by Lynch et al. (1977); series B was carried out using two versions of the apparatus shown in Figure 1 and described in the text.

\begin{tabular}{|c|c|c|c|c|c|}
\hline Series & Exp. & Pen." & Neurons & Quant. & $\begin{array}{l}\text { Comp. } \\
\text { Runs }\end{array}$ \\
\hline \multirow[t]{9}{*}{ A } & 74-R & 14 & 127 & 47 & 163 \\
\hline & $74-\mathrm{L}$ & 2 & 25 & 5 & 15 \\
\hline & $75-\mathrm{R}$ & 17 & 178 & 65 & 294 \\
\hline & $75-\mathrm{L}$ & 8 & 77 & 38 & 183 \\
\hline & 76-R & 5 & 63 & 29 & 80 \\
\hline & 76-L & 7 & 68 & 18 & 43 \\
\hline & $80-\mathrm{R}$ & 11 & 78 & 41 & 125 \\
\hline & $80-\mathrm{L}$ & 8 & 92 & 32 & 96 \\
\hline & & $(72)$ & (708) & $(275)$ & (999) \\
\hline \multirow[t]{13}{*}{ B } & 81-R & 14 & 106 & 59 & 309 \\
\hline & $81-\mathrm{L}$ & 13 & 65 & 45 & 254 \\
\hline & $82-\mathrm{R}$ & 14 & 78 & 45 & 212 \\
\hline & $82-\mathrm{L}$ & 9 & 71 & 35 & 217 \\
\hline & $83-\mathrm{R}$ & 8 & 56 & 31 & 119 \\
\hline & 83-L & 14 & 111 & 50 & 209 \\
\hline & $84-\mathrm{R}$ & 5 & 20 & 15 & 52 \\
\hline & $84-\mathrm{L}$ & 9 & 80 & 44 & 196 \\
\hline & $86-\mathrm{R}$ & 10 & 73 & 36 & 231 \\
\hline & $86-\mathrm{L}$ & 14 & 165 & 73 & 289 \\
\hline & $87-\mathrm{R}$ & 10 & 63 & 23 & 150 \\
\hline & $87-\mathrm{L}$ & 12 & 86 & 73 & 382 \\
\hline & $20 \mathrm{Hem}$. & 204 & 1682 & 804 & 3619 \\
\hline
\end{tabular}

"The abbreviations used are: Pen., microelectrode penetrations; Hem., hemispheres; Quant., neurons studied in computer-controlled behavioral runs; Comp. runs, total number of such runs. ment was replaced by a single screen measuring $100^{\circ} \times$ $100^{\circ}$, with the same back projection systems. The animal was placed either 34 or $57 \mathrm{~cm}$ from the screen (see Fig. 1).

In each of the experiments of series $\mathrm{B}$, luminous test stimuli were back-projected onto the screen. The test stimulus could be varied in size from a point source to $25^{\circ} \times 25^{\circ}$. It was usually presented at intensities between 0.2 and $0.6 \log$ unit above background, but stimuli up to 2 log units were occasionally used. The projector consisted of a single tungsten source whose light output was collimated, directed through a fast electromagnetic shutter and a photographic slide or slit aperture, reflected off mirror galvanometers and focused on the back of the screen. Program control of the shutter and galvanometers allowed generation of stimulus movements in either direction along any axis at speeds from 0 to $800^{\circ} / \mathrm{sec}$. Neutral density filters were used to control stimulus illumination levels; the background illumination of the

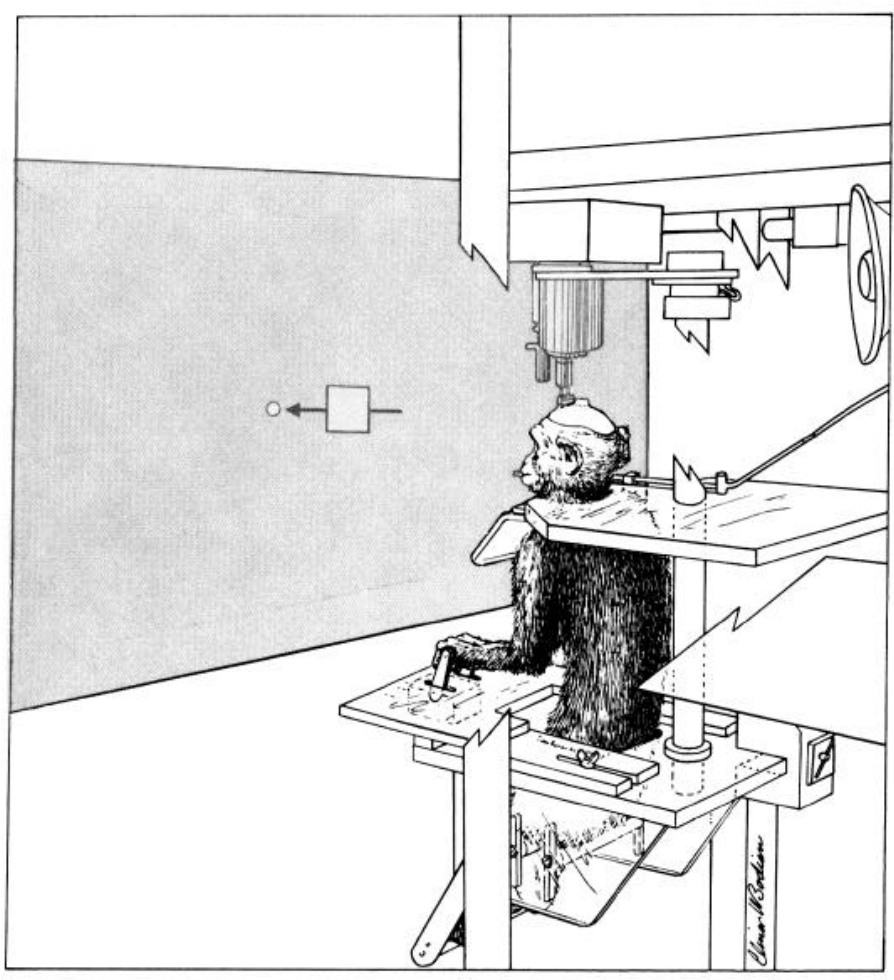

Figure 1. Behavioral task-test stimulus apparatus used for monkeys 84,86 , and 87 of series $\mathrm{B}$ of the present experiments (Table I). The head holder allowed positioning in the neutral position shown or at $60^{\circ}$ left or right. The head could be freed in the horizontal plane for periods of rest during recording sessions. Microdrive and chamber are not shown. Two back projectors were used. The first positioned the target laser spot at any locus desired and moved in any direction at velocities up to $800^{\circ} / \mathrm{sec}$. The second projected light stimuli of variable sizes, intensities, and directions of movement over the same velocity range. A panel of $600 \mathrm{LEDs}$, on 2-cm centers, could be inserted in front of the screen along guide rails. For monkeys 81 to 84 , the monkey faced a screen of three panels, each $60^{\circ} \times 60^{\circ}$; the two lateral panels were angled at $60^{\circ}$ to the central one. Projected images could be delivered to either lateral panel as the animal faced either dead ahead or toward one or the other lateral panel. Thus, the entire visual field could be examined over $180^{\circ}$ side to side. In this arrangement, the LED panel could be inserted to cover the front panel. 
screens was balanced and could be varied from complete darkness to $2 \mathrm{~cd} / \mathrm{m}^{2}$. The entire behavioral apparatus was enclosed in a light-proof chamber.

In addition, a $25 \times 27$ matrix of LEDs on 2 -cm centers could be positioned 34 to $50 \mathrm{~cm}$ in front of the monkey in each of the two screen arrangements used. The individual LEDs were under program control and could be turned on, off, or sequenced in any pattern, except that only two LEDs could be on simultaneously. A combination of normal and infrared light video monitor systems allowed observation of the monkey and the projection screens during the experiment.

The sequencing of stimulus and behavioral events and the collection and storage of neural activity and EOG records of eye position were controlled by a PDP $11 / 20$ computer. Interspike interval durations were measured to the nearest $0.1 \mathrm{msec}$.

Methods of recording. The preparation of the animal for recording sessions and the method of microelectrode recording in waking monkeys were described in a previous paper (Mountcastle et al., 1975). Each daily recording session lasted about $6 \mathrm{hr}$ during which usually one transdural electrode penetration was made. Monkeys initiated between 1000 and 2000 behavioral trials during this period, working to receive their daily water requirement.

Fye position was recorded using $\mathrm{Ag}-\mathrm{AgCl}$ cup electrodes implanted in the bones of the orbital rims with the leads running subcutaneously to a connector mounted in the acrylic head cap. Electrode pairs were selected for an offset of less than $0.2 \mathrm{mV}$, measured in $0.9 \%$ saline. Slight errors in the attempted orthogonal placement of the electrode pairs across the orbits were electronically corrected to provide true vertical and horizontal eye position records. The eye position records were sampled at rates between 20 and $200 / \mathrm{sec}$ depending upon the visuomotor task being studied.

Identification of neurons. The electrical signs of the impulse discharges of single neurons were recorded using glass-coated, platinum-iridium microelectrodes passed through the intact dura. The action potentials thought to be generated by the same single cell were identified by criteria described earlier (Mountcastle et al., 1975). We attempted in the present experiments to study neurons activated by light stimuli, per se, and we frequently terminated penetrations in which neurons with other sets of functional properties were identified. We first used qualitative methods of identification for each neuron brought under study, including manually presented visual stimuli, naturally evoked reaching and manipulation, passive somatic sensory stimulation, etc., and then initiated computer-controlled "diagnostic" runs in which a variety of visual and visuomotor tests were delivered. Further detailed and more quantitative studies of cells determined to be light sensitive were then executed using previously arranged combinations of behavioral and stimulus conditions (control sets); other control sets specifying different conditions could be generated ad hoc in a short time.

A computer run consisted of a set of up to 10 different classes, each a particular combination of behavioral and stimulus events; different classes were presented in a randomized order. The stability of the cell's response during long periods of recording was checked by repeat- ing some classes during different sequential runs. Impulse data were stored, sorted on line according to class, and displayed in a raster format on a storage oscilloscope. A printed copy of this display was made by a Tektronix 4631 unit to allow run comparisons. Continuous records of eye position and impulse activity were displayed on other oscilloscopes, digitalized, and stored.

Conventional spike train analysis techniques were used off-line for analysis of the time relations of behavioral, cellular, and eye movement events. Data summaries were generated using incremental plotter and graphics lineprinter facilities.

Anatomical studies. Small electrolytic lesions (4 $\mu \mathrm{A}$ for $4 \mathrm{sec}$, tip positive) were made at the depth of some penetrations. At the conclusion of each experiment, animals were sacrificed with an overdose of sodium pentobarbital. Small guide wires were then inserted into each hemisphere, marking a line for blocking perpendicular to the intraparietal sulcus and normal to the cortical surface. Brains were removed and placed in formalin. The fixed brains were later embedded in celoidin and sectioned serially at $20 \mu \mathrm{m}$; every section was mounted and stained with thionine.

\section{Results}

\section{General Description}

\section{The data base}

Observations were made on 20 hemispheres in two series; data summaries are given in Tables I and II. The two series differed only in the test apparatus used, as described above. We averaged 10 successful microelectrode penetrations per hemisphere. A total of 1682 neurons were brought under observation, an average of 8 cells per penetration. A neuron entered this population if its action potential was recorded in isolation from those of others for a time sufficient to allow qualitative tests aimed at identifying its functional properties. Presumptive identifications led to further studies with control sets chosen to confirm or deny them and to elucidate further the properties of the neurons. Under these latter conditions, 804 neurons were studied. We tried to isolate action potentials in the initially negative configuration because of the damage that usually accompanies close approach of the electrode tip to the cell and the inversion of the initial sign of the action potential to positive. Indeed, we commonly observed changes in the dynamic functional properties of parietal neurons to accompany spike inversion. Eight-four percent of the neurons were studied while discharging initially negative action potentials.

Location of recordings. The locations of the microelectrode penetrations made into the inferior parietal lobules of 18 of the 20 hemispheres studied are shown in Figure 2. The penetrations were located in the intermediate and posteromedial parts of area 7 in a region termed by some investigators 7 -a; we have not made a general survey of the entire inferior parietal lobule. Many penetrations close to the intraparietal sulcus passed down the cortex of its posterior bank parallel to the cortical layers, but we have not explored the sulcus to its depths. Solid circles in Figure 2 indicate penetrations in which any light-sensitive neurons were identified; in the large ma- 

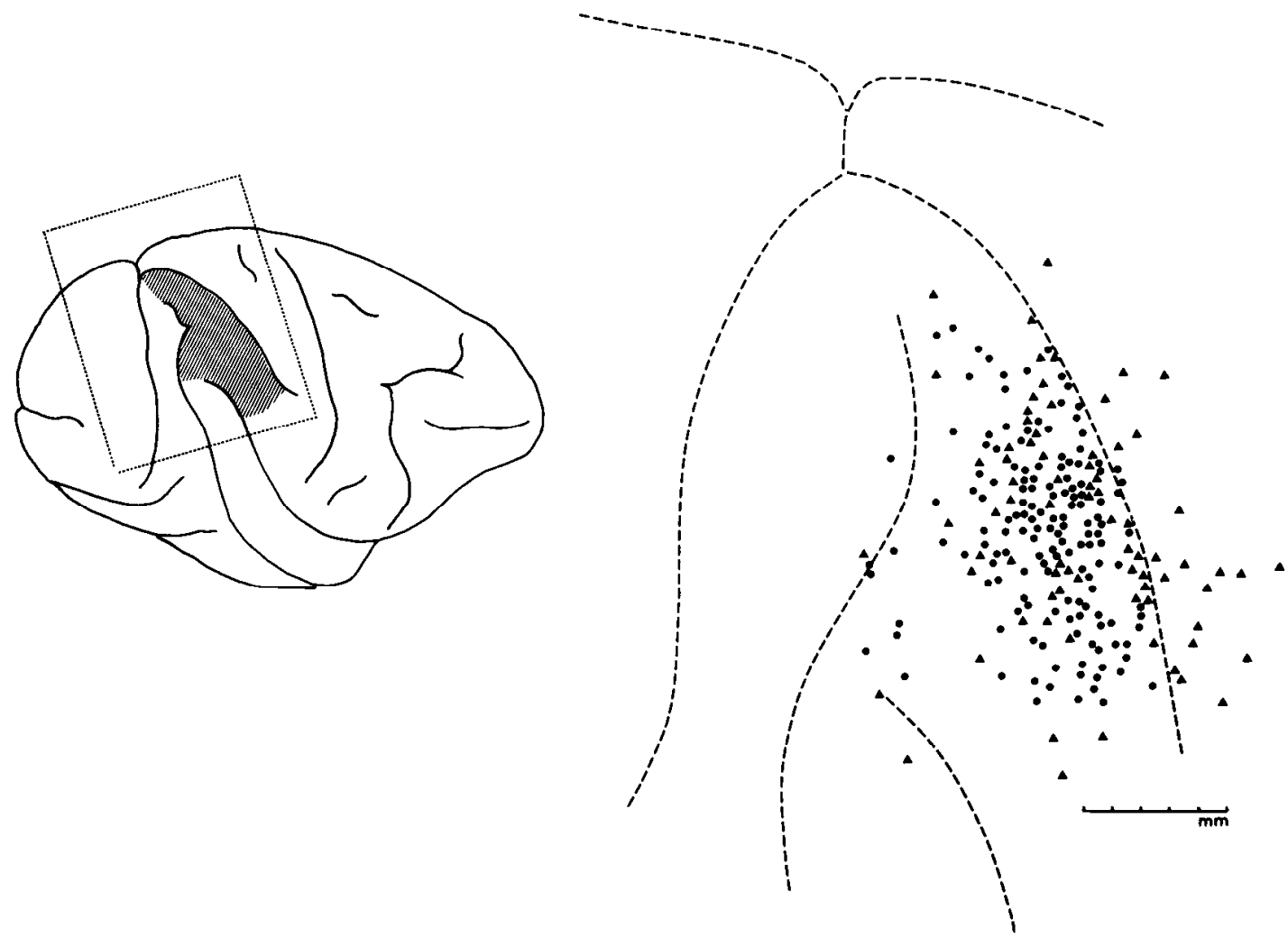

Figure 2. Locations of microelectrode penetrations. The drawing to the left shows the lateral surface of the macaque cerebral hemisphere. The area outlined is enlarged to the right, which is an average drawing of the sulcal patterns of 18 of the 20 hemispheres studied. The location of each penetration is indicated on this drawing. Penetrations marked with solid circles are those in which light-sensitive neurons were observed. Neurons of other classes (see Table II) were also encountered in many of these penetrations, often in the en bloc distribution compatible with columnar organization. Penetrations marked with solid triangles contained no light-sensitive neurons, though in many of them cells of different classes, other than light-sensitive, were frequently encountered in en bloc distribution. All penetrations made in front of the intraparietal fissure contained some neurons activated by passive somesthetic stimulation; many also contained cells of the reach-manipulation class, but no light-sensitive cells. No cells activated by passive somesthetic stimulation were identified posterior to the intraparietal sulcus in this portion of area 7-a. The error in positioning a given penetration on such an averaged diagram is 1 to $2 \mathrm{~mm}$. The results indicate that in this portion of the inferior parietal lobule, there is no clear areal segregation of the groups of cells with different properties. The data presented here reveal nothing concerning the detailed functional organization of this region, which is unknown.

jority of them, other classes of neurons were also identified, commonly in blocks with abrupt change from a zone containing cells of one class to a zone with cells of another, in the general mode of columnar organization. Triangles indicate penetrations in which no light-sensitive cells were identified; in each of them, the en bloc changes from one class to those of another were also common. No neurons activated by passive stimulation of the skin or deep tissues were identified in this region of area 7-a; many such neurons have been described by Hyvarinen and Shelepin (1979) more laterally in area 7b. We identified no light-sensitive neurons in any of the penetrations made anterior to the intraparietal sulcus.

We made electrolytic lesions in some successful penetrations in early experiments of this series. An analysis of neuronal properties in terms of cytoarchitecture and layer was not our purpose, however, and the results of the studies of the dynamic properties of parietal neurons led us to omit lesions in many penetrations. We observed that even small lesions produce marked changes in the dynamic properties of neurons within a radius of 2 to 3 $\mathrm{mm}$ of surrounding cortex and that these changes may last for several days.

\section{The classification of neurons in combined behavioral- electrophysiological experiments}

No consistent set of rules has evolved for naming the classes of neurons with different properties that can be identified in the parietal cortex in waking monkeys. This is because the activity of many parietal cells is preferentially related to one or another complex behavioral act; they are not "sensory" or "motor" as cells of the somatic sensory and the motor cortex clearly are. We have therefore labeled parietal neurons by simple empirical correlations between cell activity and behavioral event or, where it is effective, sensory stimulation.

Upon isolation of each action potential, we began a systematic qualitative examination of its properties by evoking reaching movements toward and manipulation of objects; by eliciting oculomotor events such as fixation, tracking, and saccading; by delivering visual stimuli projected upon or moved across the tangent screen as the animal fixated a stationary target; and by passive manipulation of hands and arms. In many cases, identifications made in this way were then confirmed in controlled behavioral task-test stimulus runs. In other cases, equally 
strong qualitative identifications could be neither confirmed nor proven false in any of the repertoire of behavioral tasks that we could present. In still others, presumptive identifications made in qualitative examinations were shown in controlled runs to be erroneous. Finally, many neurons were observed for which no clear identification was possible using either mode of examination. We conclude that it is not possible to establish with certainty the functional properties of any class of parietal neurons by qualitative ("clinical") examination of a waking monkey.

\section{Definition of Classes of Neurons of the Inferior Parietal Lobule}

The classes of parietal neurons that we have identified in the present series of experiments are listed in Table II and are defined as follows.

Unidentified cells $(518,31 \%)$. No clear identification could be made for 518 neurons, even though 149 of these were studied in controlled behavioral task-test stimulus runs. Neurons that we could not identify did not differ from those that we could by location in any particular part of area 7-a, by restriction to any particular depth of the cortex, or by a particular pattern of discharge. Like other classes of cortical cells, they were frequently observed sequentially in depth, forming blocks in conformity with the mode of columnar organization. However, unidentifiable cells were also observed in the midst of traverses in which other cells were clearly identified. We do not believe that difficulty in identification was caused by local cell damage, for $81 \%$ of the unidentified cells discharged initially negative action potentials, compared with $84 \%$ of the identified population. We conclude that there exists a substantial class of cells in area 7-a whose activity is unrelated to the behavioral tasks or test stimulus situations that we could deliver in these experiments; nor were these cells affected by any qualitative test that we could devise.

Fixation (218) and fixation suppression (48) cells. We identified 218 cells of the fixation class previously described by a number of investigators (Hyvarinen and
Poranen, 1974; Lynch et al., 1973a, b; Mountcastle et al., 1975; Sakata et al., 1977, 1980). We have confirmed that the activity of these cells is incremented when the animal fixates an object of a rewarding nature, a novel one, or, as Rolls et al. (1979) observed, an object which the animal regards as aversive. The second property that we take as required for identification is insensitivity to light stimuli. All cells with any response to light stimuli of any kind we have classified as light-sensitive cells in one or another of the subclasses shown in Table II. Fixation cells subtend limited gaze fields often located in the contralateral hemifield, confined to its upper or lower quadrants, or, less commonly, to the ipsilateral half of the visual field. The gaze fields of these neurons have been studied recently in a quantitative way by Sakata et al. (1980); we confirmed their observations. A subset of the fixation cells (48 in the present series) is suppressed during fixation; they too are insensitive to light stimuli.

Light-sensitive cells $(529,31 \%)$. We identified 462 neurons of area 7-a sensitive to stationary and/or moving light stimuli delivered to the visual fields during active fixations of small target lights. The visual properties of these cells are described in the following sections of this paper. A smaller group of light-sensitive cells (67) showed other properties as well: 47 were active during fixation of targets, not necessarily within their response areas, and 13 were suppressed by fixation of such targets. Seven other neurons were active during hand manipulation in total darkness, were insensitive to passive mechanical stimulation of hand or arm, and were related to visual response areas located in the lower quadrants of the visual fields.

Oculomotor cells $(163,10 \%)$. We have identified four subclasses of neurons in area 7 that are preferentially active in relation to eye movements. "Saccade neurons" $(n=60)$ are active before and during visually evoked but not spontaneous saccades to targets that do not themselves evoke responses when presented as nontarget visual stimuli, are insensitive to other visual stimuli, and are not active during tracking or fixation. Many light-sensitive cells are also influenced by saccadic movements; e.g.,

TABLE II

The classes of neurons identified in the present series of experiments in area 7-a of the inferior parietal lobule

The classification strategy and the identification parameters used are described in the text. The differences in the proportions of neurons in each class between the total number and the number studied in computer-controlled runs results from our desire to study light-sensitive (LS) neurons in this set of experiments.

\begin{tabular}{|c|c|c|c|c|c|}
\hline & & \multicolumn{2}{|c|}{ Total } & \multicolumn{2}{|c|}{ Studied in Computer Runs } \\
\hline & & No. & $\%$ & No. & $\%$ \\
\hline Unidentified neurons & & 518 & 31 & 149 & 19 \\
\hline Fixation (218) and fixation suppression (48) & & 266 & 16 & 139 & 17 \\
\hline LS only & 462 & & & & \\
\hline Fixation + LS & 47 & & & & \\
\hline Fixation suppression + LS & 13 & & & & \\
\hline Tracking & 23 & & & & \\
\hline Saccade & 60 & & & & \\
\hline Re-fixation & 25 & & & & \\
\hline Vergence & 55 & & & & \\
\hline \multirow[t]{2}{*}{ Projection (126) and manipulation (80) } & & $\underline{206}$ & 12 & 29 & 1 \\
\hline & & $\overline{1682}$ & 100 & 804 & 100 \\
\hline
\end{tabular}


their responses to visual stimuli may be enhanced or suppressed when those stimuli become targets for saccadic movements (Goldberg and Robinson, 1977; Robinson et al., 1978; Yin and Mountcastle, 1977). There appears to exist a spectrum of cells ranging from those that are activated by light stimuli and unaffected by eye movements, through those with combined properties, to others active with saccades and insensitive to light stimuli. This suggests the possibility that cells with such a gradient change in functional properties may be arranged in a sequential processing chain, but no direct evidence that this is so exists.

"Re-fixation neurons" (25) are active after completion of a visually evoked saccadic movement to a particular zone of the visual field. They are insensitive to light stimuli, are not active during casual fixations in any areas of the visual field, and thus differ from fixation neurons by the requirement for a preceding visually evoked saccadic movement. They differ from saccade neurons only in the relation of the time of discharge to eye movement.

"Tracking neurons" (23) are rare in the region of area 7 which we have studied in these experiments. They are active during slow pursuit movements, are markedly directional in nature, and are frequently suppressed during tracking in the opposite direction (Mountcastle et al., 1975). They are insensitive to light stimuli and are inactive during steadily maintained fixation. Tracking neurons are more common in the posterior part of area 7 , particularly in the anterior bank of the superior temporal sulcus. They have been studied in detail by Sakata et al. (1978). Many of these tracking neurons have recently been shown to have vestibular inputs (Kawano et al., 1980).

A newly identified subclass of neurons in area 7 we label "vergence neurons" (55) and tentatively classify them with the oculomotor group. Vergence neurons are active during saccadic or tracking movements evoked by visual targets rapidly displaced or moving slowly in the sagittal plane. Each is preferentially active with movements either toward or away from the face, but not both; they are insensitive to light stimuli. It is likely that a considerable number of neurons of the fixation class, described above, have three-dimensional fixation fields, like those that we tentatively call vergence neurons, for we have not tested them in the third dimension. Neurons with these depth characteristics have been identified in area 7 and studied by Sakata et al. $(1977,1980)$.

Projection and manipulation cells $(206,12 \%)$. Neurons of the first group (126) are active when the animal projects his arm toward a target, those of the second (80) when he manipulates within a small enclosure to obtain the target. We have confirmed our earlier observations that cells of this class are sensitive neither to passive mechanical stimulation of the hands or arms nor to visual stimuli.

\section{On naming and numbers in studies of the homotypical cerebral cortex in waking monkeys}

It is clear that the identification and classification of neurons in the parietal lobe is a difficult task and that the classes identified and the proportions of each differ between investigations. This is so because ( $a$ ) qualitative identification of cell types by simple examination cannot be made with certainty in waking monkeys, $(b)$ the controlled behavioral task-test stimulus sets that can be delivered in any particular series of experiments are limited by the test apparatus used, and $(c)$ the objectives of different investigators differ and will bias the sample of neurons observed. Table III shows that this is true for three successive studies of the inferior parietal lobule from a single laboratory, namely, our own. The three sets of experiments were made using a different test apparatus in each and with different behavioral task-test stimulus control sets. Study I was a general survey of both areas 5 and 7 , in which we did not tabulate the "unidentified cells," and concentrated on study of the fixation and projection-manipulation neurons. In study II, we did tabulate unidentified cells, but not the light-sensitive cells, and concentrated on study of the fixation and oculomotor cells. In study III (the present one), we attempted to classify all neurons observed, but have concentrated on detailed studies of the light-sensitive cells. We conclude from this experience, and from the published studies of others, that differences between cell types observed and the proportions in each, in studies from different laboratories, are due to differences in

TABLE III

The classifications of neurons of the inferior parietal lobule as identified in three successive studies

Each investigation had different objectives and was made using different behavioral task and test stimulus apparatus. Study I is from Mountcastle et al. (1975); study II from Lynch et al. (1977); study III is the present investigation.

\begin{tabular}{|c|c|c|c|c|c|c|c|c|}
\hline \multirow[t]{2}{*}{ Class } & \multicolumn{2}{|c|}{ Study I } & \multicolumn{2}{|c|}{ Study II } & \multicolumn{2}{|c|}{ Study III } & \multicolumn{2}{|c|}{ Total } \\
\hline & No. & $\%$ & No. & $\%$ & No. & $\%$ & No. & $\%$ \\
\hline Unidentified & & & 369 & 29 & 518 & 31 & 887 & 25 \\
\hline Fixation $^{b}$ & 155 & 33 & 521 & 40 & 266 & 16 & 942 & 27 \\
\hline Light sensitive & 73 & 16 & \multicolumn{2}{|c|}{ NT } & 529 & 31 & 602 & 18 \\
\hline Reach and manipulation & 128 & 28 & 136 & 11 & 206 & 12 & 470 & 14 \\
\hline Special & 21 & 4 & 32 & 3 & $={ }^{d}$ & - & 53 & 2 \\
\hline
\end{tabular}

\footnotetext{
"NT, not tabulated.

${ }^{b}$ Includes fixation suppression cells.

'Includes tracking, saccade, re-fixation, and vergence cells.

${ }^{d}$ In this study, special cells were classified as subgroups of the light-sensitive class as shown in Table II.
} 
experimental design and objective and provide no ground for polemic controversy (Robinson et al., 1978).

\section{Response of Light-sensitive Neurons to Stationary Stimuli}

\section{The response patterns}

The light-sensitive cells of area 7-a respond to stationary light flashes in the variety of discharge patterns shown in Figures 3, 4, and 5. A total of 357 cells were tested with stationary stimuli; $17 \%$ responded with sustained discharges (Figs. $3 A$, 5, and $6 C$ ); 52\% responded transiently to stimuli delivered anywhere within their response areas, and a few $(n=12)$ of these discharged a second transient at light-off. The remaining $31 \%$ responded too weakly for classification. Sixty-eight cells were suppressed by stimuli at some location, and 12 were suppressed only (Fig. $9 E$ ) and not activated by stimuli at any locations.

Many LS neurons responded vigorously to small weak lights, e.g., to the $0.3^{\circ}$ LEDs used in series A (see Figs. $3 A$ and 5). Other neurons responded only to larger stimuli. We examined the requirement for spatial summation in 11 neurons, in the paradigm of series $B$, using stimuli that were varied from 1 to $200 \mathrm{deg}^{2}$. Nine of the 11 reached maximal response with stimuli of 25 to $35 \mathrm{deg}^{2}$.
We conclude that the $6^{\circ} \times 6^{\circ}$ square or the $5^{\circ}$ diameter circle used in series $B$ saturated the requirement for spatial summation of most LS neurons.

We tested the responses of 31 LS neurons to stationary light flashes in both light and darkness. The background light condition was between 1 and $2 \mathrm{~cd} / \mathrm{m}^{2} ; 2$ min adaptation time was allowed before each test in darkness. The change from light to darkness caused no change in the response of 17 cells, an increase for eight and a decrease for three. For three other cells, we observed a reversal from an "off" to an "on" response.

The latencies of the responses of LS neurons to stationary flashes at the most sensitive loci in their response areas varied from 50 to $290 \mathrm{msec}$, with a mean of 118 msec ( $\mathrm{SD}=47 \mathrm{msec}, n=96)$ and a modal value of 110 msec. The response latency usually increased as the response intensity decreased with shifts in stimulus location within the response area.

\section{Response areas of light-sensitive neurons determined with stationary stimuli: The phenomenon of foveal sparing}

The response areas of LS neurons were determined in runs in which single stimuli were delivered during 2- to 5 -sec periods of sustained fixation. Stimuli in successive
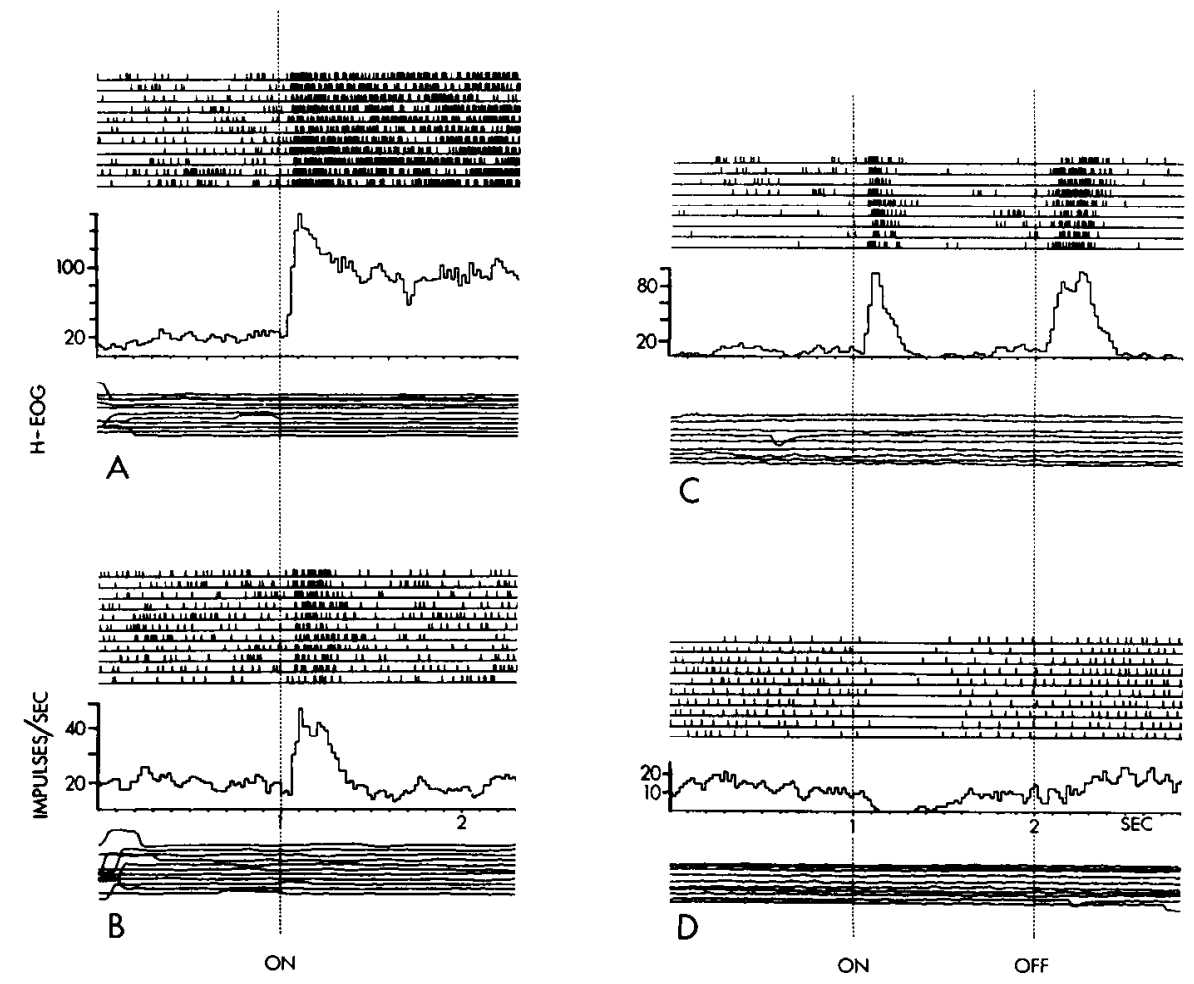

Figure 3. Types of responses of light-sensitive neurons of area 7-a to light stimuli. For each panel, and for many other illustrations that follow, each line of the upper row of records represents the time of a single behavioral trial, each upstroke the instant at which the neuron under study discharged an impulse. The histograms immediately below average these discharges. The lower records are electro-oculograms, one for each of the trials shown above. Vertical dashed lines indicate in sequence on and, for some runs, off of the light stimulus. $A$, Discharge pattern of a neuron responding to a LED stimulus, placed within its response area, with a sustained discharge; the stimulus and the discharge continued beyond the length of the record shown here. $B$, Discharge pattern of a neuron responding to a similar LED stimulus with a transient discharge. $C$, The on-off discharge pattern of a neuron elicited by a $6^{\circ} \times 6^{\circ}$ square stimulus placed in its response area. $D$, This neuron responded to a similar stimulus with a cessation of discharge. Response type $B$ is the most common; types $C$ and $D$ are relatively rare. Range of stimulus intensive, 0.5 to $1 \mathrm{log}$ unit above background. Time intervals, $100 \mathrm{msec}$. 

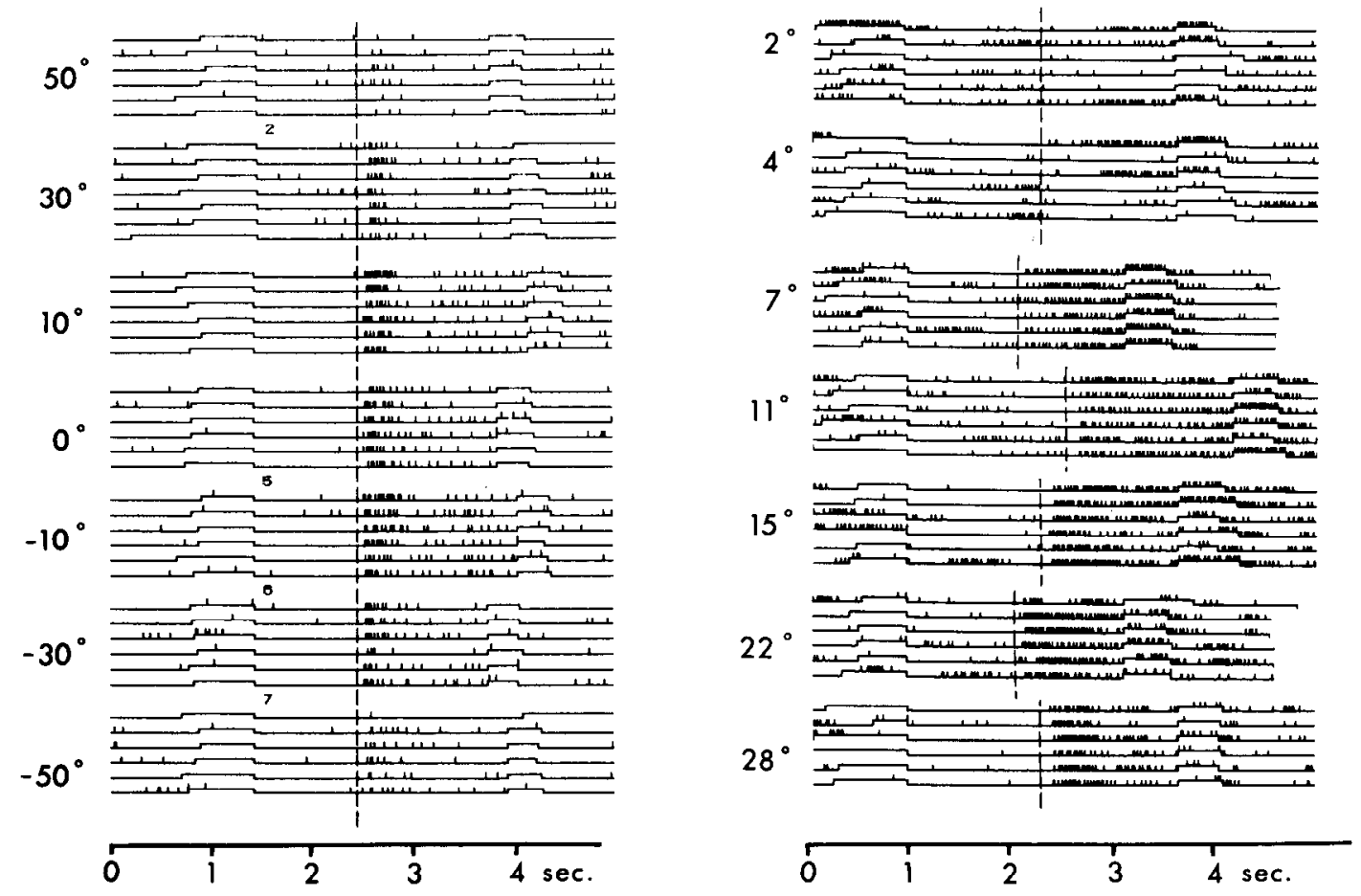

Figure 4. Illustration of the two major classes of response areas of parietal light-sensitive neurons. The impulse replicas to the left were obtained in the study of a neuron typical of those with foveal response areas. The maximal response was evoked by stimuli at or close to the central line of vision, and stimuli placed $m$ either side along the horizontal meridian evoked decreasing responses in a nearly symmetrical pattern. Test stimulus $6^{\circ} \times 6^{\circ}$ : syuare, about $5 \mathrm{~cd} / \mathrm{m}^{2}$, delivered against a background of about $1 \mathrm{~cd} / \mathrm{m}^{2}$. In each record, the first step displacement indicates target light on, the second the animal's closure of the signal key, the third the dimming of the target light, the fourth the instant of key release by the animal. The vertical dashed lines indicate onset of the light stimulus, which remained on for $1 \mathrm{sec}$. Records to the right are those of a neuron related to a response area confined to the contralateral hemifield but which spared the foveal region; the test stimulus was an LED physically identical to the target LED which evoked no response at all. Test stimuli were delivered to the locations indicated, along the contralateral horizontal meridian; no responses were evoked by it when placed at similar locations on the ipsilateral side. All stimuli were delivered as the animal held steady fixation of the target light placed dead ahead.

trials of a run were presented in a pseudorandom sequence at different positions along axes passing through the fixation point. Histograms were constructed for the neural activity evoked by the stimuli at each position.

There are three salient features of these response areas: They are, with few exceptions, very large as compared with those of neurons of either the striate cortex or the superior colliculus; they are frequently bilateral; and many display the characteristic of foveal sparing. The last refers to two observations. Firstly, LS cells rarely (i.e., only eight neurons) respond to the foveally fixated stimulus target even though physically identical stimuli may evoke vigorous responses when delivered close to the fixation point (see Fig. 5). Secondly, perifoveal zones of insensitivity can be demonstrated using other images projected during steady fixation of the target light. Foveal sparing is illustrated further by the discharge replicas of Figure 4 (right) and the polar plots of Figure $7, A, B$, and $C$.

We have observed that even a large stimulus $\left(6^{\circ} \times 6^{\circ}\right)$ centered on the fixation point will not evoke a response in about half of the light-sensitive cells. A simple classification of response areas based on this criterion is given in Table IV which shows that even after excluding those neurons that responded to large stimuli centered on the fixation point, but not to the fixation light itself, $127 / 216$
$=59 \%$ of the response areas spared the region of the fixation point. Samples of the response areas of cells related to contralateral response areas with foveal sparing are given in Figure 8, and samples of those related to bilateral response areas with foveal sparing are given in Figure $9, A, B, C$, and $D$. For some neurons of the latter group, the central zone of inexcitability appeared as a hole in an otherwise continuous response area; in others, it separated two disjointed response areas, one in each half of the visual fields. The examples given in Figure 9 show that the zone of foveal sparing may be large $(A)$, may separate two response areas that extend symmetrically to $50^{\circ}$ or more into each of the visual half-fields $(B)$, may be confined to a narrow zone around the point of fixation $(C)$, or may separate zones of unequal sizes $(D)$.

The phenomenon of foveal sparing raises the question of whether it results from a dynamic influence of the act of target fixation upon the excitability of the systems linking retina to the parietal lobe, or whether the distribution of response areas can be accounted for on the basis of a connectivity that for this class of LS neurons precisely exempts the point of fixation. We emphasize that the response areas described were determined as the animal fixated a central target, dead ahead, for we have observed two powerful effects that facilitate the re- 


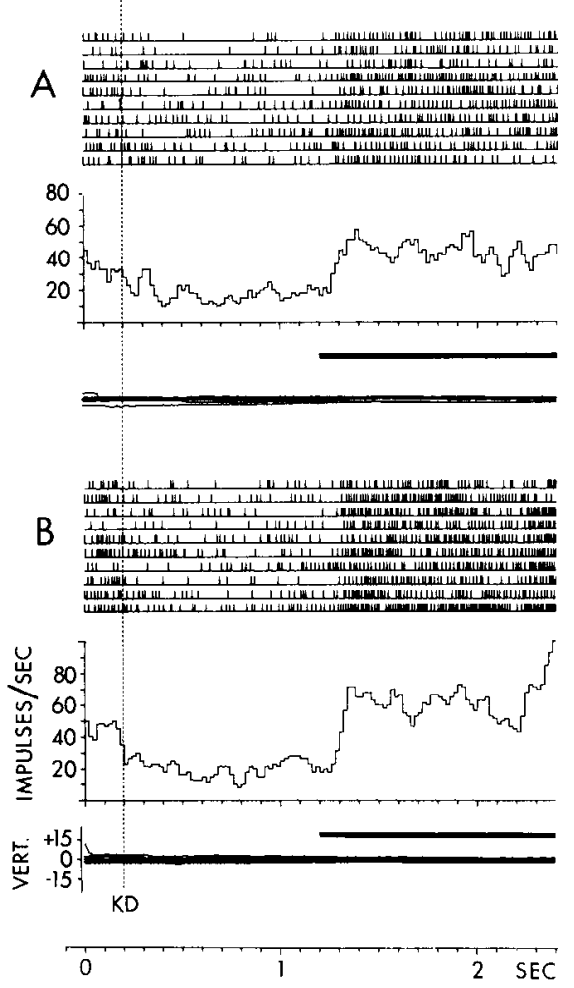

Figure 5. The phenomenon of foveal sparing. Records of a neuron related to a bilateral response area with foveal sparing. The set of records above are of responses evoked by an LED light placed $3^{\circ}$ above the fixation point along the vertical meridian, those below by a similar light $3^{\circ}$ below the fixation point. Each trial was initiated by the monkey at $K D$ (keydown), the vertical dashed line, after which he steadily fixated the target light for $1 \mathrm{sec}$ before the test stimulus was delivered; this target light evoked no response of the neuron. The test stimuli were delivered during the times marked by the solid bars; each evoked an intense and sustained cellular discharge. The lowest records of each panel are superimposed vertical electro-oculograms, one for each of the trials illustrated above. They show the steadily maintained fixations throughout the trials. Time intervals, $100 \mathrm{msec}$.

sponses of LS neurons to light stimuli delivered in extrafoveal locations: the act of target fixation and the angle of gaze. We will describe them in detail in later papers.

The second class of response areas of parietal LS neurons we term foveal-inclusive $(89 / 216=41 \%)$, because for them, $6^{\circ} \times 6^{\circ}$ stimuli superimposed over the fixation point did evoke responses. A number of these foveal-inclusive neurons are most sensitive to stimuli placed at the fixation point itself, and the response intensity for them declined more or less symmetrically on either side (Fig. 4, left). The response areas of these cells are almost reciprocal in their spatial distributions to many of the cells with bilateral fields and foveal sparing. Figure 10 shows that the response areas of foveal-inclusive neurons may vary from small $(B)$ to very large and may extend to over the $100^{\circ}$ examined along an axis $(D)$. For other neurons of this class, the response areas were asymmetrically placed, as shown by the polar plot of Figure $10 E$ and the histograms of Figure $6 B$. Response areas confined to the ipsilateral visual field are extremely rare.

We observed for all except eight of the cells which we have classified as foveal-inclusive that even when a $6^{\circ}$ $\times 6^{\circ}$ stimulus centered over the fixation point evoked an intense response, fixation of the target light itself evoked nothing (see Fig. 4, left). The question then is whether foveal sparing exists also for some of the neurons which we have classified as foveal-inclusive, because of the response to large stimuli, or whether the absence of response to the fixation target light reveals only a requirement for spatial summation. We have not resolved that problem and thus in parsimony, have classified these neurons as having foveal response areas.

\section{The Responses of Light-sensitive Neurons to Moving Stimuli}

The LS neurons of area 7-a are particularly sensitive to moving as compared to stationary stimuli in both the intensity of the response and the size and distribution of the response areas. We chose for the majority of our studies to move lights along the axes of the visual fields that cross the fixation point; we define as inward movements toward the fixation point while those in the opposite direction are considered outward. We have studied a smaller number of neurons with stimuli moving along other axes, but we have not used movements along axes centered upon and rotated about the "center" of the response areas, for the latter are large and possess no readily defined centers.

Figure 11 shows two common features of the lightsensitive cells of area 7-a. They are much more sensitive to moving than to stationary light stimuli, and they are, in the majority of cases, differentially sensitive to the direction of stimulus movement. We now wish to document these properties further and to describe the relation to each other of the directional vectors in different sectors of the visual fields.

\section{Sensitivity to the direction of movement}

We studied $172 \mathrm{LS}$ neurons in paradigms resembling that of Figure 11; the results are summarized in Table V. Each of these cells was tested with standard light stimuli moving along the horizontal and vertical meridians in both directions. Many cells were also tested along diagonal axes and some along axes that did not intersect the fixation point. Table $\mathrm{V}$ shows that $90 \%$ of the neurons tested were directionally sensitive in at least one halfsector of either the vertical or the horizontal meridian. The varieties and the degrees of this directionality are illustrated by the sets of histograms of Figure 12. These results, together with those of Figures 13, 14, and 15, are typical of all our observations. We conclude from them that the large majority of LS neurons of area 7-a are sensitive to the direction of stimulus movement and that for most of them, this directionality is very strong. Stimuli moving in a direction opposite to that which excites may elicit suppression of on-going activity for a few cells, but more commonly has no effect. Although some of the histograms of Figure 12 suggest that a response area plotted with moving stimuli may in some cases overlap the fixation point, we believe that for most this is not 

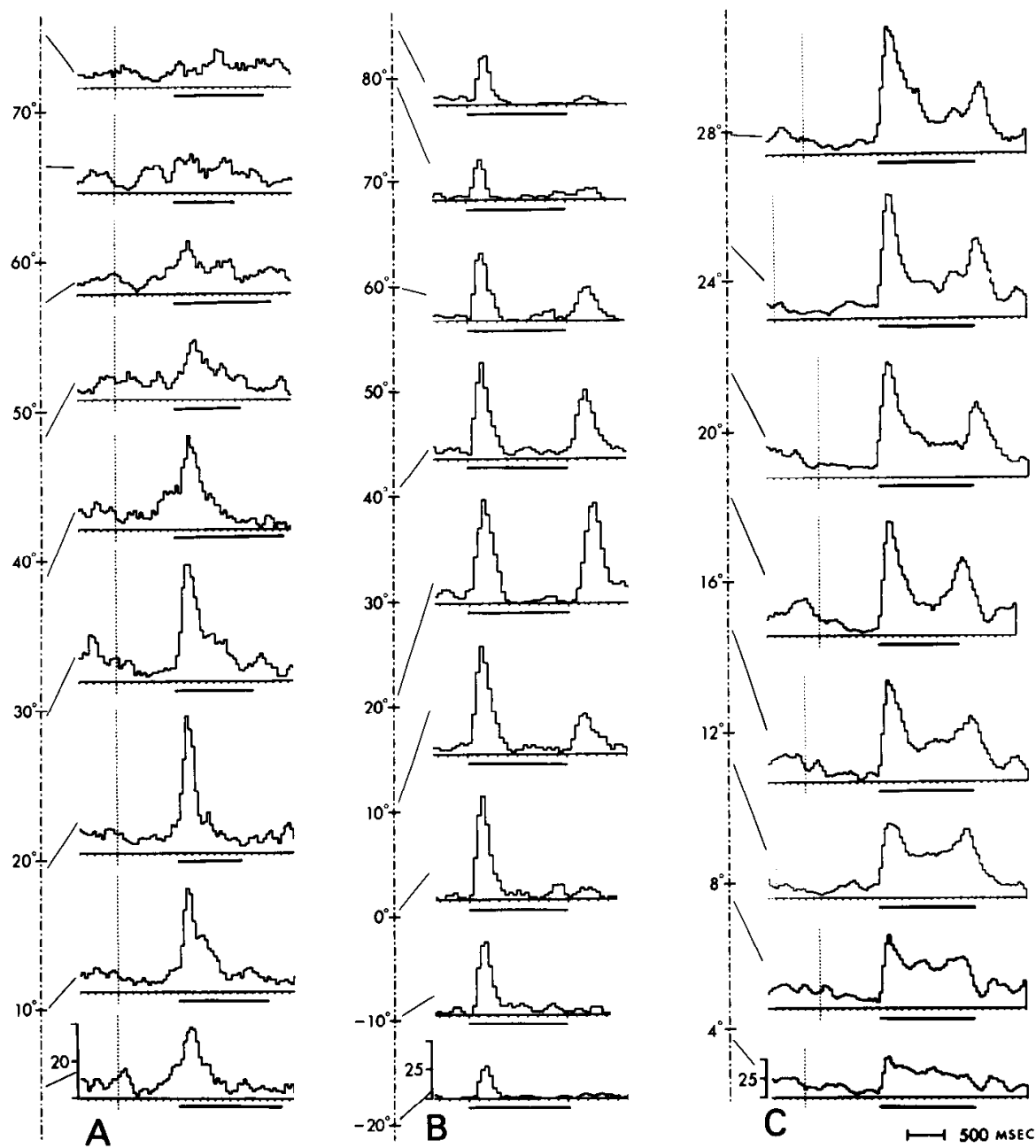

Figure 6. The response areas of three light-sensitive neurons of area 7 -a. In each case, the histograms sum the responses of a neuron to 6 to 10 trials of stimuli delivered at each of the locations indicated along the meridian of the visual fields that transected the longest axis of the response area. The solid bars indicate the stimulus periods. $A$, Results for a neuron activated from the contralateral visual field with foveal sparing. Vertical dashed line indicates switch closure by the animal after which he steadily fixed a target LED dead ahead; this visual stimulus to the foveal region of the retina evoked no response. Then, a physically identical LED came on at one of the locations indicated along the contralateral horizontal meridian. It evoked transient discharges when within an area extending from within $5^{\circ}$ of the central line of gaze to at least $60^{\circ}$ laterally into the contralateral visual field. $B$, Study of a neuron related to a bilateral, eccentrically distributed response area that included the fovea. Here the trials have begun before the records do; fixation of the target light evoked no response, but the $6^{\circ} \times 6^{\circ}$ square light (intensity about 1 log unit above background) evoked an on-off discharge at locations from ipsilateral $20^{\circ}$ to at least contralateral $85^{\circ}$ along the horizontal meridian. $C$, Study of a neuron that responded with a sustained discharge under circumstances similar to those of $A$. Again, the onset target light evoked no response, and the field extended from as near to the fixation point as $4^{\circ}$, outward along the horizontal meridian, contralaterally; it was tested only to $28^{\circ}$. Study $B$ presents an unresolved question: Here the target light evoked no response, but the $6^{\circ} \times 6^{\circ}$ square centered on the fixation point did so (see record labeled $0^{\circ}$ ). We have for reasons of parsimony classified all such neurons as related to foveal response areas, though more detailed studies with small stimuli have revealed for some cells a small zone of foveal sparing, as the absence of response to the fixation light suggests.

true, but that the overlap by the histogram is due to after-discharge. We have observed such an after-discharge of the response when inwardly moving stimuli are stopped and turned off just short of the fixation point. The majority of the movement response areas are laterally placed along the axes examined, and though they may extend close to the center of the visual field, they almost always terminate just short of the fixation point itself.

\section{Sensitivity to the velocity of movement}

We have tested the sensitivity of 70 of these parietal LS neurons to stimulus velocity over the range from 10 to $800^{\circ} / \mathrm{sec}$. Although a few cells responded over this entire range, the majority had flat velocity sensitivity functions with broad peaks in the range from 30 to $60^{\circ} \%$ sec. These preliminary studies suggest that while this set of neurons can provide very sensitive signals of stimulus 

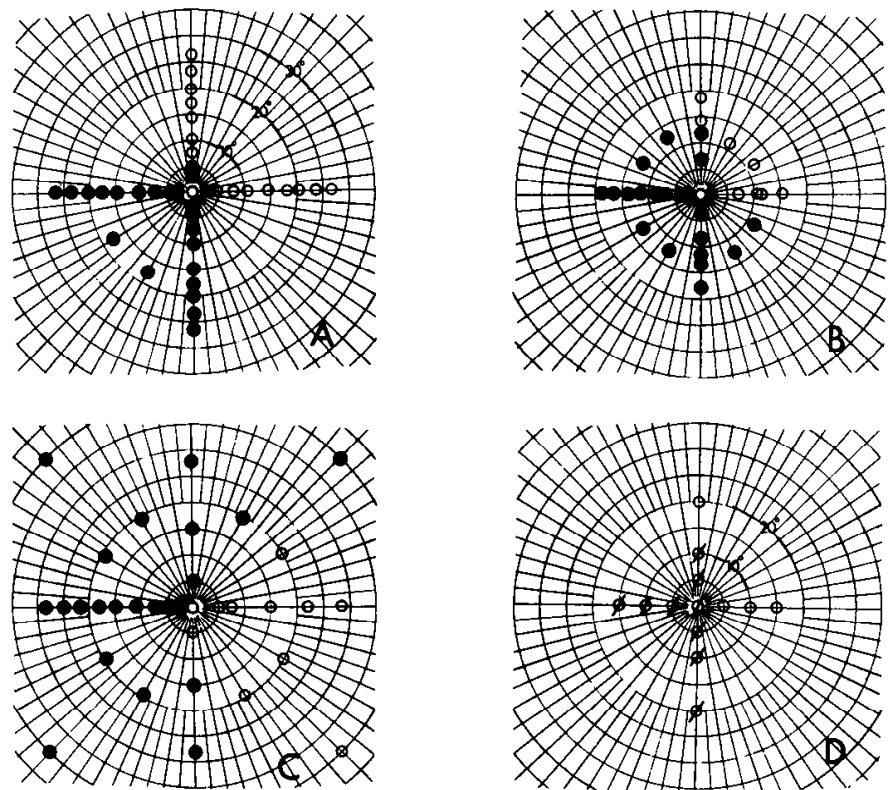

Figure 7. Polar plots of the response areas of light-sensitive neurons of the parietal cortex. Six to 12 trials were delivered at each location, and histograms were constructed summing the evoked activity; stimuli were delivered at the several locations in a pseudorandom sequence. Solid circles indicate locations at which stimuli evoked responses; open circles, those at which they did not; open circles with slash, those at which stimuli evoked suppression. In each case, the animal maintained steady fixation of a LED target light at position 0, 0 throughout each trial. For the neurons of $A, B$, and $C$, the light stimulus was a LED physically identical to the fixation target; for that of $D$, it was a $6^{\circ} \times 6^{\circ}$ square. $A, B$, and $C$ illustrate large response areas that extend into more than one-half of the visual field, but exempt the central zone of gaze. $D$ illustrates the response area of one of the rarely encountered neurons that was suppressed but never excited by the light stimulus.

movement over a wide range of velocities, it is not likely to signal accurate information concerning velocity itself.

\section{The opponent organization of vectors in the response areas of light-sensitive neurons}

We commonly observed for LS neurons with directionally sensitive response areas in three or four quadrants of the visual field that the vectors in opposite halves of the field pointed in opposite directions. We term this opponent vector organization. Of 88 cells that were related to response areas in three or four quadrants, 63 showed opponent organization orientation, and for $75 \%$ of these, the directional vectors point toward the fixation point; the remainder point away. Fourteen neurons showed identical vectorial directions in two halves of the visual field, and for 11 , the vectorial directions were mixed (Table V, I: A, B, and C). Forty-seven other directionally sensitive neurons were related to response areas in only one quadrant of the visual field, and 18 to two adjacent quadrants. No pattern of vector orientation could be defined for these neurons.

The varieties of vectorial organization that we have observed are illustrated in Figures 13, 14, and 15. Figure
TABLE IV

Distribution of the response areas of the light-sensitive neurons of area 7-a between those sparing and those including the central zones of gaze

The phenomenon of foveal spring is described in the text.

I. Response areas sparing the fovea

127

A. Unilateral areas

B. Bilateral areas

C. Foveal sparing, laterality undetermined

II. Response areas including the

fovea

A. Bilateral, symmetrical

B. Bilateral, asymmetrical

C. Bilateral, degree of symmetry undetermined

66

43

18

Total: $\overline{216}$
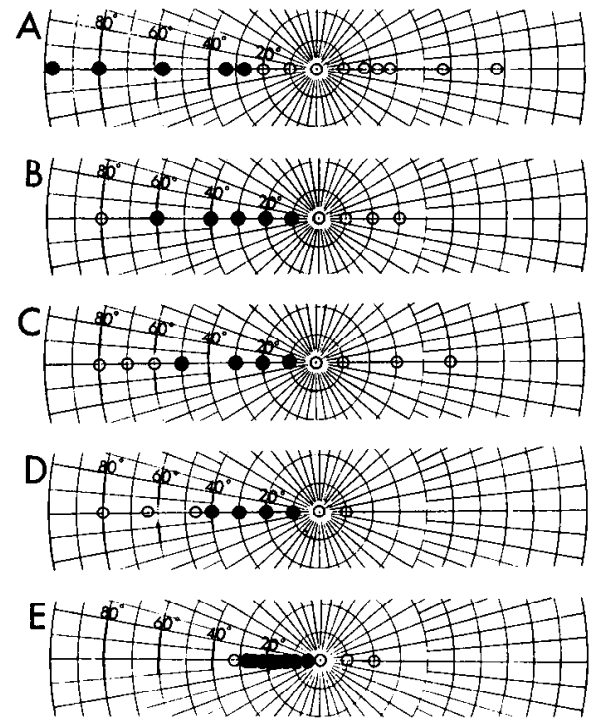

Figure 8. Polar plots of response areas of parietal lightsensitive neurons: unilateral areas with foveal sparing. The distribution of each response area along its major meridian is shown, plotted as in Figure 7. These five neurons were related to contralaterally located response areas of different sizes, with sparing of the foveal and immediately perifoveal regions. Solid circles, locations at which stimuli evoked responses; open circles, locations at which they did not.

13 , left, illustrates the directionality typical for a neuron related to a single response sector along the contralateral horizontal meridian. The histograms to the right show response properties of a neuron related to two response sectors placed along adjacent half-meridians. The directional sensitivity is strong in each of these two cases, and the vectors point inward toward the fixation point. The histograms of Figure 14 illustrate for two neurons directional sensitivity along each of the four half-meridians of the visual fields; in each, the directional vector points inward, in typical opponent fashion. An instance of fourquadrant, directional sensitivity away from the fixation point is shown by the histograms to the left in Figure 15. Finally, a rare instance of a neuron sensitive to moving lights but insensitive to their direction is given to the 

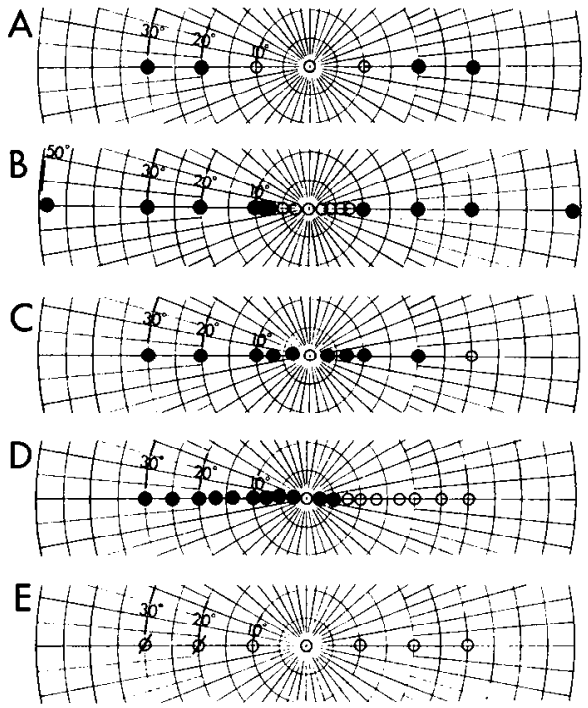

Figure 9. Polar plots of response areas of parietal lightsensitive neurons: bilateral areas with foveal sparing. The distribution of each field is shown along its major meridian, plotted as in Figure 7. The neurons of $A, B, C$, and $D$ were related to bilateral response areas that varied in extent and degree of symmetry; in each, the foveal and immediately perifoveal zones are spared. The neuron of $E$ was suppressed from a contralateral response area. Symbols as in Figure 8.
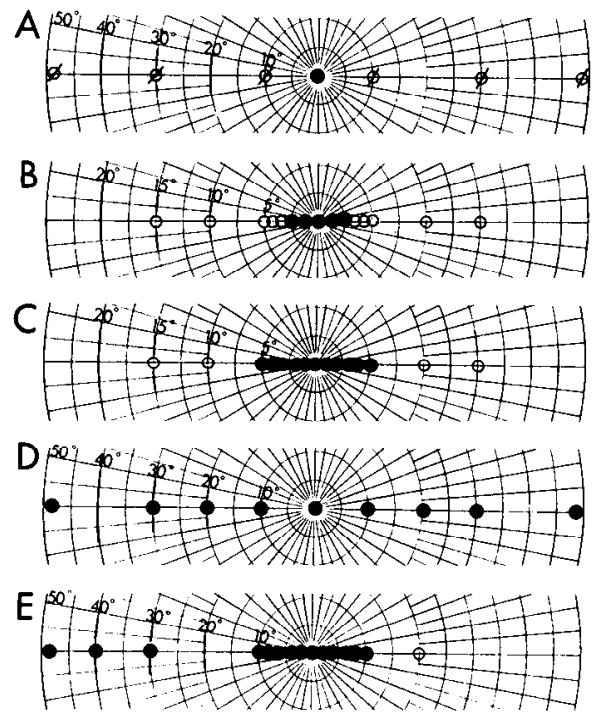

Figure 10. Polar plots of response areas of parietal lightsensitive neurons: bilateral areas including the foveal region. The extent of each area along its major meridian is shown, plotted as for Figure 7. The neurons of $B$ and $C$ were related to a symmetrical field that extended over at least $100^{\circ}$ along the horizontal meridian of the visual field; it also responded to stimuli placed over $100^{\circ}$ along the vertical meridian, not shown here. The cell of $E$ was related to a typical foveal asymmetrical field. The neuron of $A$ was the only one that we ever observed with an excitatory "center" with suppressive flanking zones. Symbols as in Figure 8.

right of Figure 15, which also illustrates that moving lights may evoke a suppression of on-going discharge as they cross the fixation point.

These and other patterns of directional sensitivity and the relation between vectors in different parts of the visual field are illustrated by the cartoons of Figure 16.

\section{Is opponent vector organization radial to the point of fixation?}

The data that we have presented indicate that many directionally sensitive LS neurons of area 7 may be activated by stimuli moving through large response areas that frequently include sectors in both the contralateral and ipsilateral visual fields crossed by the opposite halves of the major axes of the visual fields. All our observations suggest that these opposed vectors point either toward the center or outward toward the rims of the visual fields. Observations of neurons tested along four or eight axes (Fig. 16A) suggest that for many cells, the directionally opposed vectors are organized in a radial manner around the fixation point. Our findings are not sufficient to establish this point with certainty, and further evidence is needed from experiments in which directional sensitivities are measured along axes that do not intersect the fixation point. Some observations which we made on a few cells are of interest in this regard, for in them, we found vectors pointing toward a meridian; for example, with horizontal vectors aimed toward the vertical meridian at several different vertical levels (Fig. 17). What is certain from our observations is the opponent vector mode of organization, which often occurs in a radial manner around the fixation point, although other forms of organization of the opponent fields exist also.

Comparison of the response areas of light-sensitive neurons determined with stationary and with moving stimuli

We examined 91 light-sensitive neurons to determine the correspondence between their response areas determined with stationary and with moving stiumli. We found a clear mismatch both for size and location for 61 of those cells (67\%). This mismatch may be marked, as illustrated in Figure 11 for a neuron directionally sensitive to moving stimuli, and relatively insensitive to stationary ones. An equal lack of correspondence in the reverse direction has been observed for a few cells, and the LS neurons of area 7-a appear to vary over a wide spectrum in regard to the degree of this incongruence.

\section{The relation between excitatory and suppressive response areas}

We observed $68 \mathrm{LS}$ cells (13\%) whose discharge rates were suppressed by light stimuli. For 12 of these, suppression was the only change evoked by lights placed anywhere in the visual fields, while the remainder were related to a mixture of suppressive and excitatory zones within their total response areas. Moving stimuli in some part of their response areas suppressed the discharge of 54 of the 68 cells, and the majority of these were sensitive to the direction of movement. However, only nine cells were excited by moving stimuli in one direction of movement and suppressed by the reciprocal movement in the same region of the response area. For the remainder, the suppressive and excitatory zones were spatially separate, but no regular relation between the two was established. Figure $10 \mathrm{~A}$ illustrates the only neuron studied that was 

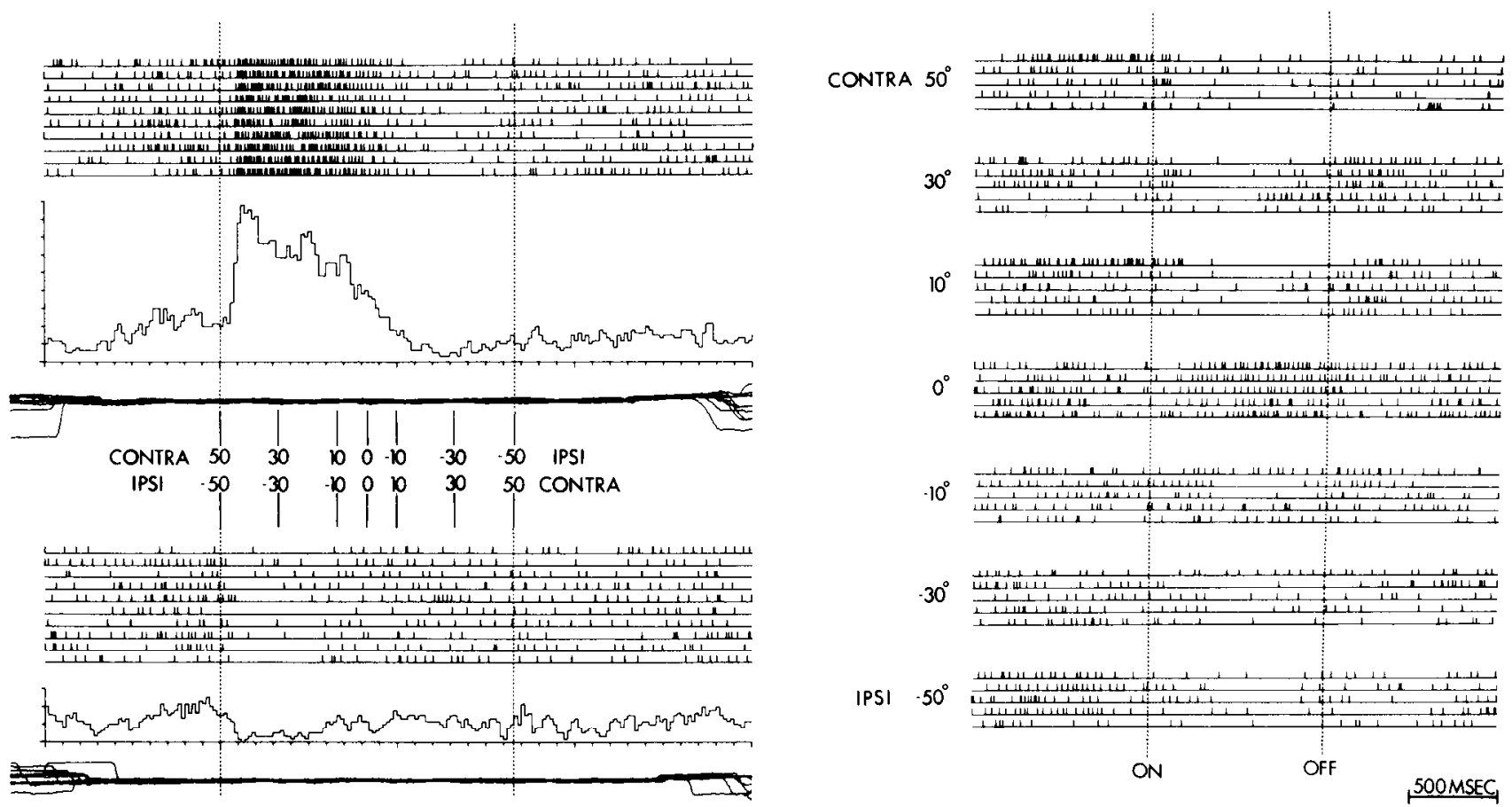

Figure 11. Directional sensitivity and opponent organization. The impulse replicas, histogram, and superimposed electrooculograms shown at upper left illustrate the intense response of this neuron elicited by the stimulus as it moved from a locus $50^{\circ}$ contralaterally (CONTRA) to one $50^{\circ}$ ipsilaterally (IPSI) along the horizontal meridian at $60^{\circ} / \mathrm{sec}$. The records below show the result when the stimulus moved in the reciprocal direction. The neuron was differentially sensitive to the direction of movement in the contralateral field; movements in either direction in the ipsilateral half elicited a mild suppression. The sets of records to the right show that physically identical but stationary stimuli elicited little or no change from any of seven locations along the same axis tested with moving stimuli. Stimulus was a $6^{\circ} \times 6^{\circ}$ square, about 1 log unit above background.

TABLE V

Distribution of the light-sensitive neurons of area 7 -a sensitive to moving stimuli, in regard to the property of directionality

The phenomenon of opponent vector organization is described in the text.

I. Directionally sensitive neurons 153

A. With opponent vector organi63 zation

B. Without opponent vector organization

C. With mixed organization

D. Single sector, directional

E. Two adjacent sectors, directional

II. Directionally insensitive neurons

Total: $\frac{19}{172}$

related to an excitatory area with flanking suppressive zones on either side.

\section{Discussion}

We first summarize the functional properties of the light-sensitive cells of the inferior parietal lobule (IPL) and the anatomical pathways that link the retina to this cortical region. We then consider to what extent the salient features of parietal LS neurons, foveal sparing and opponent vector organization, can be accounted for by the functional properties of neurons in those parts of the striate and collicular visual systems known to project upon the IPL and whether further processing with elab- oration of these features must be assumed to occur within the parietal cortex itself.

Studies of humans and other primates with parietal lobe lesions have revealed a number of complex defects and abnormalities in visual behavior that may occur without changes in visual acuity and with intact visual fields. We discuss what contributions the afferent visual signals to and the processing of them within the IPL, revealed in the functional properties of the light-sensitive celis, may make to these and other complex aspects of visual behavior. These are the direction of attention, especially visual attention; the perception of the spatial relation of objects; and the perception of movement, especially of objects moving in the peripheral fields during fixation, including the apparent movement of the stationary environment during locomotion with eyes fixed on the horizon.

\section{The functional properties of parietal light-sensitive neurons}

The LS cells of area 7-a respond to stationary flashing lights with sustained, transient, or on-off discharge patterns. They are related to large response areas in the contralateral half-fields, and two-thirds of these areas extend to include what are usually smaller regions of the ipsilateral visual field (Table IV, IB and II). Fifty-eight percent of all fields, whether uni- or bilateral, do not include the region at and immediately surrounding the fixation point (Table IV, I). This central region of unresponsiveness is frequently quite small, e.g., 3 to $6^{\circ}$, compared to the total area of the bilateral response area 

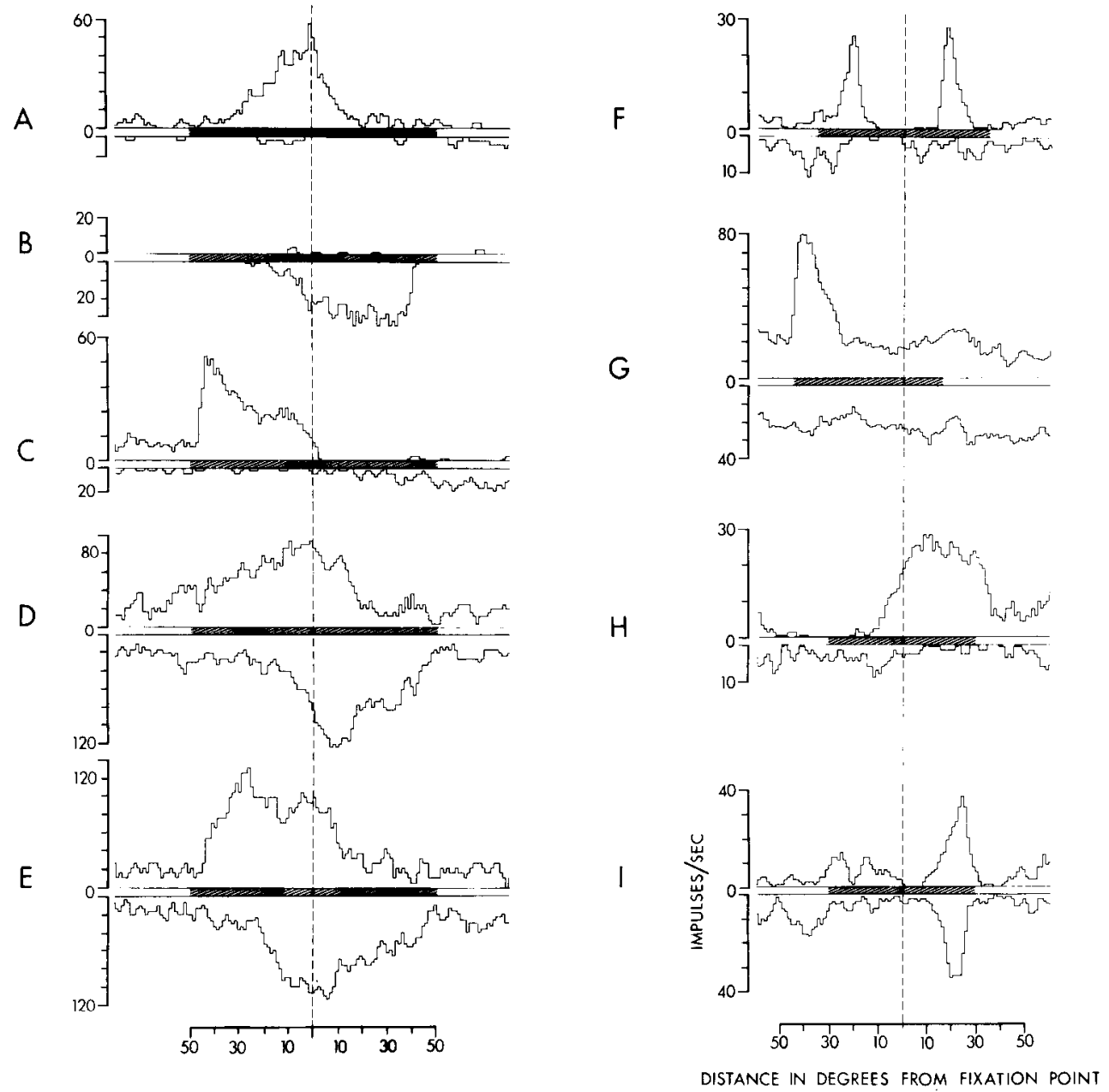

Figure 12. Varieties and degree of directional sensitivity of parietal light-sensitive neurons. The upper histogram of each pair sums the discharges of a neuron evoked by a stimulus moving for $100^{\circ}$ in one direction along either the vertical or horizontal meridian, whichever was the major axis of the response area. The lower histogram of each pair sums the discharges evoked by the stimulus moving in the reciprocal direction, along the same axis. The lower histogram is reversed in direction in regard to time, so that the spatial coordinate for the two histograms is identical, as shown by the scales at the bottom. The neurons of $A$, $B, C, G$, and $H$ show an absolute directionality, with response areas confined to one-half of the visual field. The small overlap of the fixation point by the histograms of $A, B, C$, and $H$ is thought to be due to after-discharge. $D$ and $E$ are histogram pairs for the same neuron, $D$ for movements along the horizontal meridian, $E$ the vertical. The response area for this neuron was very large and included the fixation point; the directional sensitivity was weak, which produced only a skewing of the histograms. $F$ shows results for an unusual neuron related to two areas sensitive to the same direction of movement, symmetrically placed in either visual half-field. $I$ shows the result for one of the small number of neurons without directional sensitivity. Stimuli were $6^{\circ} \times 6^{\circ}$ squares or $5^{\circ}$ circles, about 1 log unit above background, moving at either 30 or $60^{\circ} / \mathrm{sec}$. Shaded bars, segments of the meridians transitted by the stimuli.

disposed around it. The response areas of the remaining $42 \%$ of LS cells do include the fovea, at least when neurons that respond to a $6^{\circ} \times 6^{\circ}$ light centered on the fixation point are included. All except eight of these neurons did not respond to the fixation light itself, so that the proportion of LS neurons with foveal sparing may actually be much larger than Table IV indicales. The response areas of a significant number of fovealinclusive neurons are symmetrically arranged around the point of fixation, yield maximal responses to stimuli centered at that point, and vary in size from those $5^{\circ}$ in diameter to those that include all of the visual field tested $\left(100^{\circ}\right.$ along each meridian). Although many response areas of intermediate size were encountered, the separation into two large classes, one inclusive and one exclusive of the foveal region, seems clear. We observed that a number of neurons tested with stationary stimuli were suppressed by lights in some areas of the visual fields, but no regular relation between excitatory and suppressive zones has been defined. A few neurons were suppressed and never activated by any stationary stimuli. We have not observed orientation sensitivity, but we have not tested with bars longer than $10^{\circ}$.

Parietal LS cells are sensitive to stimuli moving along the axes of the visual fields that intercept the fixation point, and many are differentially sensitive to the directions of movement along those axes. The response areas determined by moving and by stationary stimuli are frequently incongruent, though overlapping, and in the limiting cases, neurons may be insensitive to stationary 


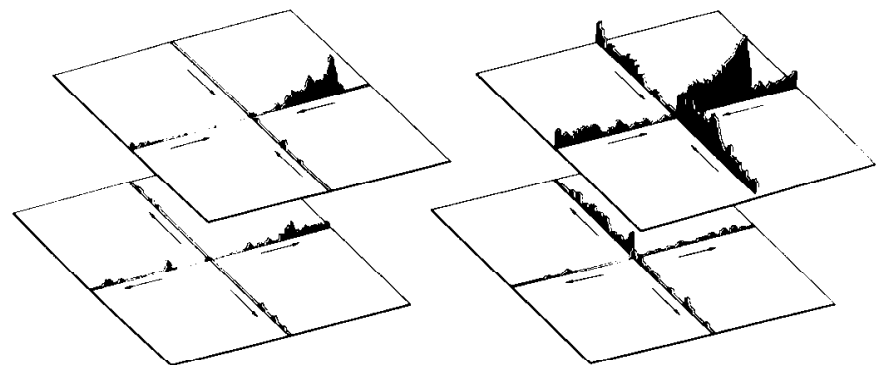

Figure 13. Directional sensitivity of parietal light-sensitive neurons. The histogram displays of this figure and those of Figures 14 and 15 were constructed in the following way. Each histogram, summarizing the discharges of a neuron occurring during movement of the light stimulus along a meridian across the visual field, was cut at the point where the stimuli crossed the fixation point. The halves of the histograms for each of the four directions of motion toward the fixation point were joined and oriented on the upper plane of each illustration; the outward motion halves similarly on the lower plane. Approximately $100^{\circ}$ of visual angle centered at the fixation point is illustrated; stimulus movements covered slightly shorter transits of the visual field. Left, histograms of the responses of a neuron related to a response area confined to one quadrant of the contralateral field, with inward directionality. Right, histograms of the response of a neuron related to two adjacent quadrants, with inward vector organization. These results, and those of Figures 14 and 15, were obtained with a stimulus of $6^{\circ}$ $\times 6^{\circ}$, about $1 \log$ unit above background, moving at $30^{\circ} / \mathrm{sec}$.

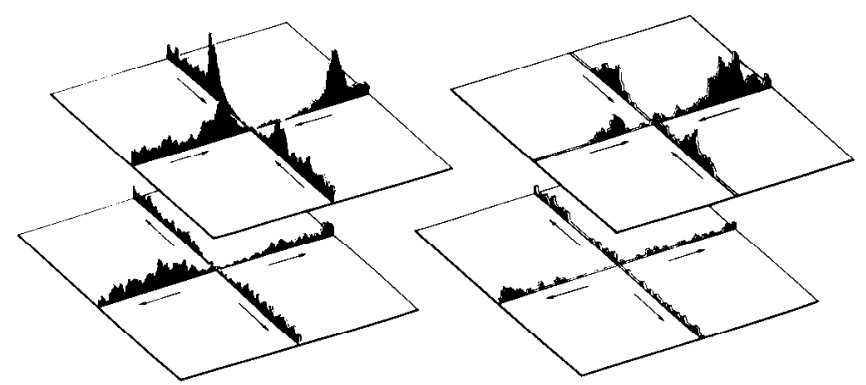

Figure 14. Directional sensitivity of parietal light-sensitive neurons, with opponent vector organization. Directional response planes constructed as described for Figure 13. Left, response histograms for a neuron related to response sectors in each of the four quadrants of the visual fields; this result is typical of inwardly directed opponent vector organization. Right, a similar result for another neuron, but here the cell is most sensitive to light moving inwardly along the contralateral horizontal meridian, a common pattern.

but respond vigorously to moving stimuli and vice versa. The movement vectors point symmetrically toward the fixation point for $75 \%$ of the neurons tested and point outward for the remainder. We term this pattern opponent vector organization. The response areas determined with moving stimuli commonly extend close to but exempt the point of fixation. We discuss below what contributions this system might make to certain forms of visual behavior.

\section{What pathways link the retina and the inferior parietal lobule?}

The cortical connections of the IPL dominate its extrinsic projections and receptions, as compared to its direct linkages to the thalamus (Divac et al., 1977; Mesulam et al., 1977; Pandya and Vignolo, 1969; Petras,
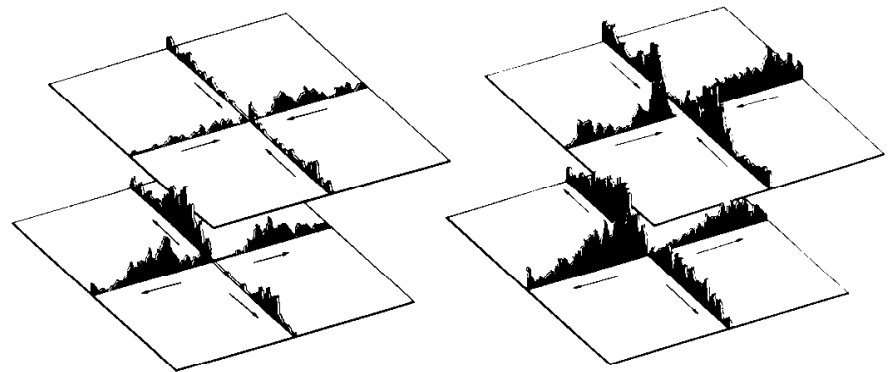

Figure 15. Directional and nondirectional sensitivity of parietal light-sensitive neurons. Directional response planes constructed as described for Figure 13. Left, response histograms for a neuron with four-quadrant outwardly directed sensitivity. Right, response histograms of a neuron sensitive to stimulus movement in each of the four quadrants, but with very weak directional sensitivity and with foveal suppression.

1971; Seltzer and Pandya, 1978). The IPL is usually divided into a posteromedial, $\mathrm{PG}$ or 7-a, and an anterolateral one, PF or 7-b (von Bonin and Bailey, 1947), but study of serial sections cut perpendicularly to the intraparietal sulcus suggests that it contains a number of fields. Indeed, Seltzer and Pandya (1980) have recently described two new subdivisions, areas POa-i and POa-e. These are long, strip-like zones in the posterior bank of the intraparietal sulcus. Area 7 has been treated as a single unit in many of the anatomical studies in which its extrinsic connections were defined. It is still uncertain whether smaller divisions within this very large cortical area entertain all or only some fraction of the total pattern of connections established for the whole. We consider here only those connections that might account for the properties of the light-sensitive neurons of the IPL.

The retino-striate system. Light-evoked activity may reach the IPL from area 17 either transcortically over a multi-step relay or from the prestriate cortex. Area 17 projects directly upon the cortex of the superior temporal sulcus (STS) (Montero, 1980; Pandya and Kuypers, 1969; Seltzer and Pandya, 1978; Ungerleider and Mishkin, 1979; Weller and Kaas, 1978; Zeki, 1974, 1976) and indirectly to that same STS target through the prestriate zones (Zeki, 1974, 1975). The IPL might then receive relayed light-evoked activity over a projection to it from the cortex lining the banks and floor of the STS. Pandya and Kuypers (1969), using an anterograde degeneration method, and both Mesulam et al. (1977) and Stanton et al. (1977), using tracer methods, have described this projection. Such a transcortical pathway was suggested by Ungerleider and Mishkin (1978) on the basis of their lesion-behavioral studies. They concluded that the spatial aspects of visual behavior measured by the landmark test, thought to depend in part upon the integrity of the IPL, require visual input from both the foveal and perifoveal field regions of area 17 and suggested that the links connecting area 17 and the IPL are transcortical. A zone of cortex within area 19 projects upon area $\mathrm{PO}-\mathrm{a}$, in the posterior bank of the intraparietal sulcus (Seltzer and Pandya, 1980) and PO-a is thought to project to other portions of area 7 .

In addition to these transcortical pathways, lightevoked neural activity may propagate along pathways linking the prestriate cortex to the IPL via a cortico- 

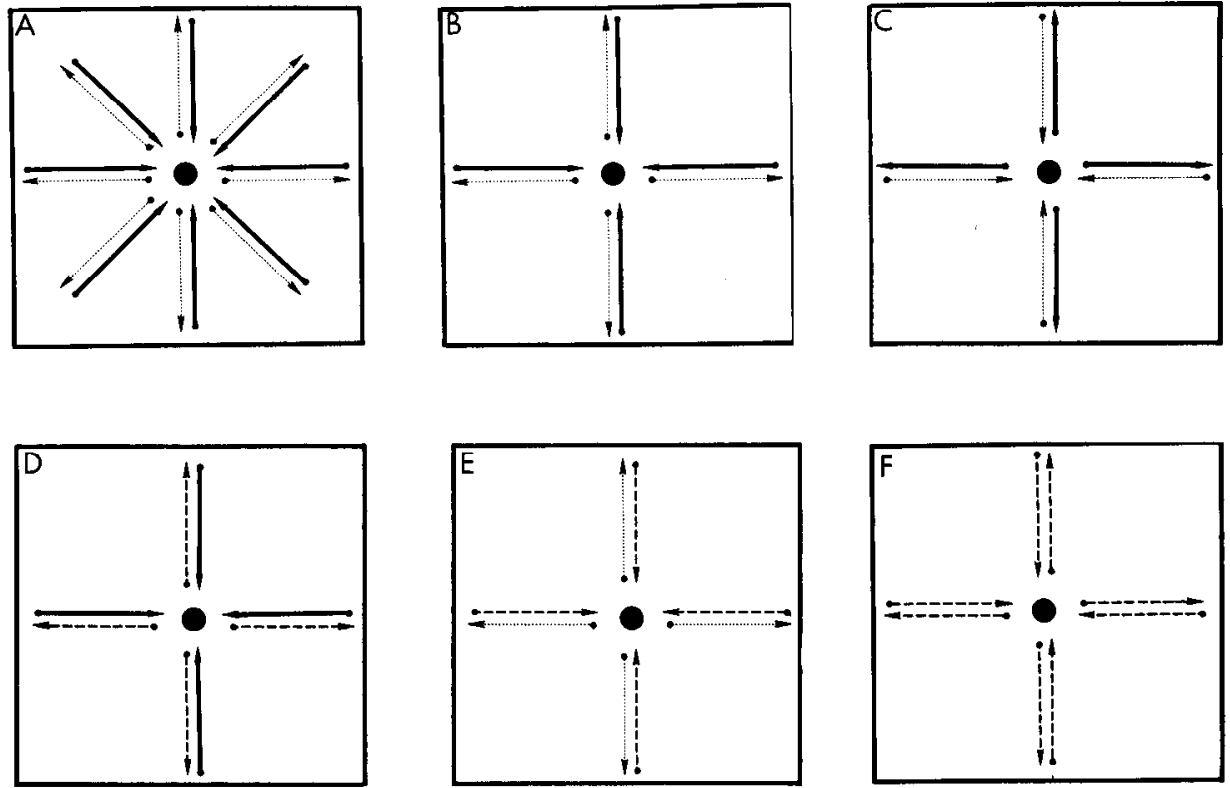

Figure 16. Varieties of the opponent vector organization of parietal light-sensitive neurons. Cartoons illustrating the variety of directional organization. The arrows indicate the direction of stimulus movement, but they are unrelated to the intensity of the response or the spatial extent of the response areas. Solid lines, excitation; dashed lines, suppression; dotted lines, no effect. $A$, typical radial opponent vector organization tested along eight axes crossing the fixation point with no reciprocal suppression. $B$ and $C$, inwardly and outwardly directed opponent vector organization tested along four axes with no reciprocal suppression; $D$, the same with reciprocal suppression in all directions tested; $E$, suppression for all inwardly moving stimuli in the pattern of opponent vector organization; $F$, suppression by movement in either direction along each of the four axes tested. Types $D$, $E$, and $F$ were observed rarely.
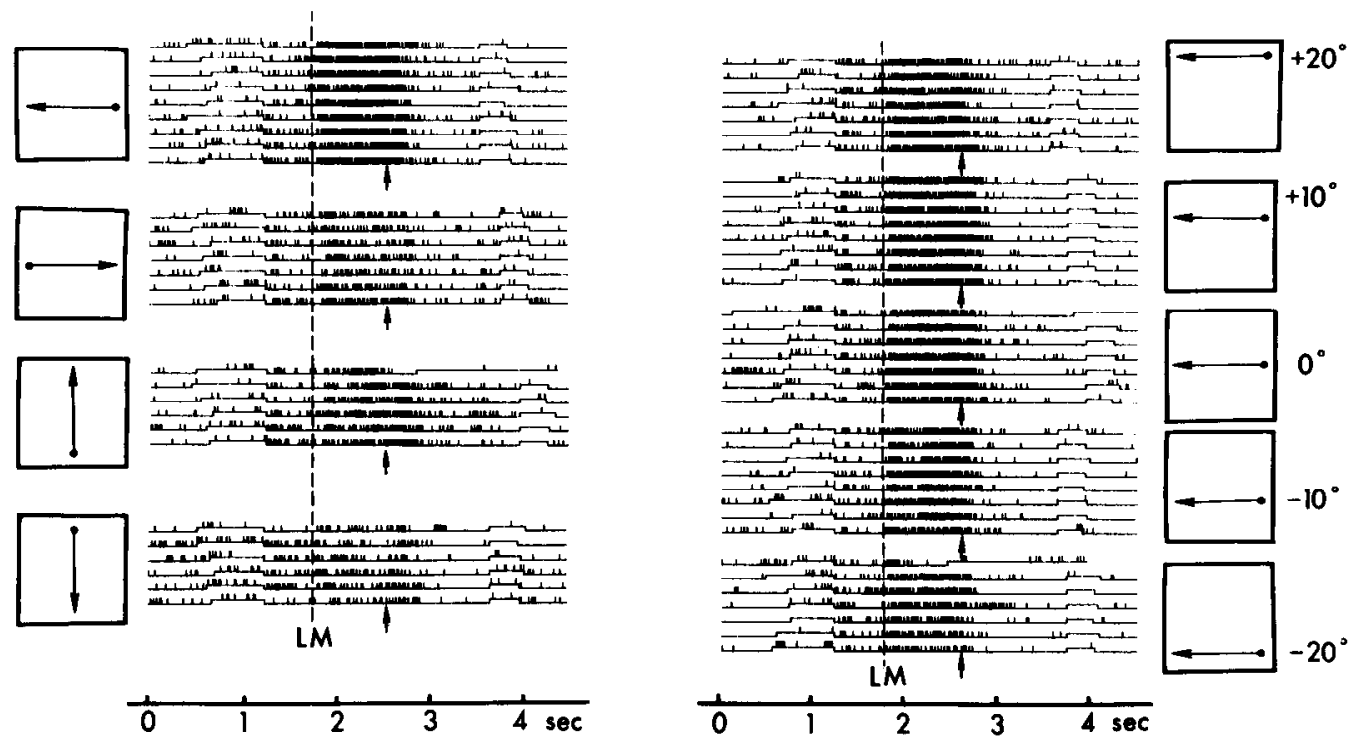

Figure 17. Nonradial opponent vector organization for a parietal light-sensitive neuron. Impulse replicas illustrated were obtained in a study of the opponent vector organization of an unusual type. The records on the left were obtained when the light stimulus moved in each direclion along the vertical and horizontal meridians as indicated. A discharge was evoked for each movement, most intense for that from right to left (contralateral to ipsilateral) and weakest for movement from above downward. The slight overlap of the fixation point by the discharge (arrows) is thought to be due to after-discharge. The records on the right were obtained when the stimulus was moved from the contralateral to the ipsilateral side in the horizontal direction, at five different vertical positions. The vector organization appears to be inward toward the vertical meridian. Less intense discharges were evoked by inward movements from the ipsilateral side, as shown by the second set of records on the left. Velocity of movement, $60^{\circ} / \mathrm{sec}$; stimulus $6^{\circ} \times 6^{\circ}$, about $1 \mathrm{log}$ unit above background; $L M$, onset of light and its movement.

thalamocortical loop. The prestriate cortex projects in a retinotopic manner upon the inferior pulvinar (PI) and the immediately adjacent region of the lateral pulvinar called PL- $\alpha$ (Benevento and Davis, 1977; Rezak and Benevento, 1977) and to the lateral posterior (LP) and lateral dorsal (LD) nuclei as well; these latter projections are only weakly retinotopic. LP and LD are major sources of thalamocortical afferents to the parietal lobe. The IPL also receives fibers from the medial pulvinar nucleus and from the intralaminar group, most densely from the paracentral nucleus (Baleydier and Mauguiere, 1977; Divac et al., 1977; Kasdon and Jacobson, 1978; Trojanowski 
and Jacobson, 1976; Pearson et al., 1978). Thus, the inferior parietal lobule may receive relayed activity from the retino-striate system over a pathway involving a multi-step transcortical path and, in addition, over the cortico-thalamocortical loops described.

The retino-collicular and pretectal systems. The topographic projection of the retina upon the superficial layers of the superior colliculus and the upward thalamocortical projections from them provide another pathway over which light-evoked activity might reach the IPL. The superficial layers of the superior colliculus receive convergent, retinotopic projections in register from both the retina and the striate cortex. They project upward upon the retinotopically organized PI and PL- $\alpha$, as well as upon a number of other thalamic nuclei (Benevento and Fallon, 1975; Benevento and Rezak, 1976, 1977; Benevento et al., 1977; Rezak and Benevento, 1975; Partlow et al., 1977). These nuclei, PI and PL- $\alpha$, project upon the striate and prestriate cortical areas but not upon the parietal lobe. The link from the prestriate cortex to the STS and thence to the IPL might complete such a convergent projection of collicular and striate systems upon the striate cortex. The deeper layers of the superior colliculus and the retinoreceptive sublentiform and olivary nuclei of the pretectal region project upon common thalamic targets: the intralaminar nuclei, PL$\beta$, and LP, nuclei that do project upon the IPL, but which have a weak if any retinotopic organization (Benevento et al., 1977; Magnuson, et al., 1979).

In summary, the inferior parietal lobule receives convergent projections from the retino-striate and retinocollicular systems, and from the pretectal nuclei as well. There are several pathways from the striate cortex to the IPL; each is characterized by a partial loss of retinotopic organization. The properties of the cells of the intermediate and deep layers of the superior colliculus, and of the two pretectal nuclei that project upon the thalamus, suggest that they may provide signals to the cortex concerning eye position and movement-and some neural activity related to the state of attention, poorly defined as that must be for the present. These two projections from two major sources are convergent with reference to the inferior parietal lobule as a whole. The possibility remains that their projections upon the IPL may be partially segregated in regard to the different zones of this large area or even in regard to more local processing units.

\section{How to account for the static properties of the light- sensitive neurons of the parietal lobe}

The large response areas of the parietal LS cells suggest a convergence and further intracortical processing of neural activity relayed from the visual system, for they are an order of magnitude or more larger than are those of neurons of the striate cortex or of the superior colliculus, in the monkey (Goldberg and Wurtz, 1972; Hubel and Wiesel, 1968, 1974; Poggio et al., 1975; Schiller et al., 1976a, b; Schiller and Koerner, 1971). There is a progressive increase in field size and loss of retinotopy at each successive step in the transcortical pathways that project upon the IPL. These properties, however, do not serve to differentiate between striate and collicular sources and may equally well be produced by a convergence within a relayed upward projection from the superior colliculus. No characteristic of either system, morcover, can account for the bilateral response areas of many parietal LS neurons. This bilaterality is most likely due to the dense interhemispheric system that links reciprocally the two inferior parietal lobules via the corpus callosum.

'The bilaterality of the visual field representation in area 7 -a resembles that of light-sensitive cells of the temporal homotypical cortex, which is known to depend upon callosal connections (Rocha-Miranda et al., 1975). There is no evidence for a direct link between the inferior temporal cortex and the IPL. Somewhat comparable bilateral response areas have been observed for a group of visually responsive cells located dorsal to the inferior temporal cortex in the floor and anterior bank of the superior temporal sulcus (Desimone and Gross, 1979). These neurons possess directional attributes resembling in some respects those of the LS cells of area 7-a; they depend upon the striate cortex for that directionality, but not for light sensitivity per se (Desimone et al., 1979). Reciprocal connectivity between this part of the superior temporal sulcus and area 7 is suggested by the findings of projections in one direction by Jones and Powell (1970) and in the other by Mesulam et al. (1977).

The response areas of foveal-inclusive parietal LS neurons may be produced by a preferential projection relayed from the foveal components of the striate system. Indeed, a foveal domination exists for some prestriate areas (Zeki, 1975) and is exaggerated even further in the striate-prestriate projection target in the posterior bank of the superior temporal sulcus (Dubner and Zeki, 1971; Zeki, 1974). Neurons with foveal-inclusive response areas are unlikely to depend upon a dominant projection from the colliculus, where the foveal representation is much less elaborate than it is in the striate system (Cynader and Berman, 1972; Goldberg and Robinson, 1978; Schiller et al., 1974).

The distribution of bilateral response areas with foveal sparing and the sharp exemption of the foveal region from many large response areas that are confined to the contralateral visual field, cannot be explained by any known properties of neurons of either the striate or the collicular systems. It seems unlikely that such a sharp separation into groups of neurons with response areas that include the fovea and those that spare it is produced by a weak retinotopic mapping in the partially shifted overlap mode. One might then expect to observe an entire spectrum of response areas gradually shifted in location across the visual fields. A large majority should then contain the representation of the foveal region because of the large sizes of the response areas. This is just the pattern observed in studies of the visually related cortex of the temporal lobe (Gross et al., 1969). A mapping function might still explain our observations on the assumption that two selective matrix transformations lead from a complete retinotopic map to sets with and without foveal sparing. Indeed, there is a selective projection from that portion of the prestriate cortex (area 19) containing the representation of the contralateral, peripheral visual field, to the posterior bank of the IPS (Seltzer and Pandya, 1980). On the assumption that this region projects in turn to the exposed surface of the 
inferior parietal lobule, a convergence within this projection could account for the contralateral fields with foveal sparing that we have observed.

Last among the hypotheses which we consider is that foveal sparing is produced by a dynamic process. The lack of response to the fixation target in all but eight of the LS neurons suggests that a dynamic process suppresses a response to the fixation target without affecting the excitability of the system to eccentrically placed targets. We have found in recent experiments that the fixation of a target raises the excitability of parietal LS neurons to eccentric targets (Motter and Mountcastle, 1980). What is less certain is whether this dynamic control process affects the central and peripheral regions of the visual fields in a differential manner-suppressing one while facilitating the other-and thus underlies the phenomenon of foveal sparing.

\section{How to account for the dynamic properties of the light- sensitive neurons of the parietal lobe}

The most striking dynamic property of parietal lightsensitive neurons is their sensitivity to stimulus movement and direction. The elaboration of these properties is a major feature of intracerebral processing of neural activity reaching the striate area from the lateral geniculate nucleus (Hubel and Wiesel, 1968; Poggio et al., 1975; Poggio and Talbot, 1980; Schiller et al., 1976a, b). Zeki has shown that these properties are further and selectively elaborated in a striate/prestriate target zone in the posterior bank of the superior temporal sulcus, a region thought to form the last step in one transcortical pathway linking the visual cortex and the inferior parietal lobule (Zeki, 1974, 1976). Directionality is an uncommon property of light-sensitive cells of the superior colliculus of the primate (Cynader and Berman, 1972; Goldberg and Robinson, 1978; Goldberg and Wurtz, 1972; Marrocco and $\mathrm{Li}, 1977$; Schiller and Koerner, 1971; Updyke, 1974). It seems likely, therefore, that the striate system is the major source of light-evoked afferent input to the directionally sensitive neurons of the parietal lobe of the monkey.

Certain of the dynamic properties of the light-sensitive neurons do not appear in any of the candidate afferent inputs to the IPL and must be attributed to intracortical processing. The first is the wide range of velocities to which parietal neurons respond (up to $800^{\circ} / \mathrm{sec}$ ). The second is the opponent organization of the movement vectors for neurons related to bilateral response areas. The first requirement for the construction of opponency in the horizontal dimension is the presence in each parietal lobe of neurons related to strictly contralateral response areas, with either inwardly or outwardly directed directional sensitivity. Numbers of cells with these properties were identified in area 7-a (Fig. 13 and Table V). The second requirement is an interhemispheric convergence of neurons with strictly contralateral fields and with identical directional sensitivities, perhaps via the corpus callosum. The target cells of this convergence, in either hemisphere, would then respond as do the parietal cells with bilateral response areas that we have observed, i.e., with sensitivity to movement in one direction in one half of the visual field and to opposite movements in the other. Opponency in the vertical direction could result from processing of afferent input within the parietal lobe itself.

In summary, it seems reasonable to attribute certain properties of the light-sensitive neurons of the inferior parietal lobule to a relayed and convergent projection of afferent signals from the striate and the collicular components of the visual system and others to further processing within the parietal cortex itself and, in addition, to the effects of a powerful state control system. These properties are the very large response areas, with and without foveal sparing; the acute sensitivity to stimulus movement over a wide range of velocities and to direction of that movement; and opponent vector organization. It remains to be determined how universal and how balanced such a putative convergence might be upon the several classes of parietal neurons with "visual" properties or, indeed, whether different functional modules of the area that contain one or another of the identified classes of parietal cells might receive projections with different degrees of dominance from striate or collicular sources. A reasonable set of working hypotheses might be these: $(a)$ that LS neurons receive visual input largely from the striate system, but perhaps not exclusively so; (b) that the several classes of oculomotor neurons receive input mainly via ascending projections originating in the intermediate and perhaps also the deeper layers of the superior colliculus and the pretectal nuclei but not exclusively so; $(c)$ that the smaller numbers of neurons that display visual as well as other properties may receive a more balanced input from the two sources, in regard to that part of their input that is visual in nature; and $(d)$ that those classes of neurons that appear to be independent of direct or closely linked afferent drive and more closely related to initiatives toward action, the fixation, reach, and manipulation classes of parietal neurons, receive no direct input originating in the visual system.

\section{Relations to behavior}

The visual stimuli that we used to determine the response areas and functional properties of parietal LS neurons were not used to initiate, guide, or terminate the behavior of the monkey subjects. On the contrary, the animals were trained to maintain fixation of a target light that did control behavior and to ignore flashing or moving test lights even when the latter crossed the point of fixation at high velocity. A break in fixation and eye movement greater than a pre-set threshold, e.g., 1 to $2^{\circ}$, during the test period was penalized as an error trial in the latter animals of the series. Thus, we do not attempt to make direct "behavioral correlations." We do address the question of which aspects of visual behavior might, by virtue of their own parametric requirements, depend, at least in part, upon a light-driven system with the properties that we have observed, particularly those classes of visual behavior and visually guided motor behavior disturbed by lesions of the parietal homotypical cortex.

We consider these candidate functions of the parietal light-sensitive system in the context of the distinction made by Trevarthen (1968) between focal and ambient vision. Focal vision is that which serves with the greatest spatial acuity refined discriminative acts in regard to the shape, size, color, etc., of objects and which has evolved 
to a high degree of proficiency in primates. Linked to it is a motor apparatus for maintaining the line of gaze upon objects of interest, for saccadic movements to new targets, and for smooth pursuit tracking of moving ones. Focal vision guides praxic operations upon objects in the local environment, particularly manual operations. There is commonly associated with focal vision a co-linear direction of visual attention, though the two can be partially dissociated under laboratory conditions (Posner et al., 1978), and certainly under natural ones, attention is sometimes shared between focal and ambient vision.

Ambient vision relates to what Trevarthen (1968) defined as behavioral space (the "immediate extrapersonal space," Mountcastle, 1976); it is a spatial frame with head as center, symmetrically distributed in the frontal plane, and polarized in the antero-posterior direction disproportionately in the forward direction. The immediate behavioral space is defined by the extent of manual operations, which may of course be extended by tools, but behavioral space obviously extends many meters to include interactions with objects and individuals. Ambient vision is the vision of relations within this space; it extends over the entire visual field but is differentially more effective in the periphery, including the monocular crescents. It operates effectively in daylight illumination and is, compared to focal vision, greatly enhanced in dim surroundings as focal vision fades. Motor operations thought to depend mainly upon ambient vision are head and body orientations, postural adjustments, and locomotory displacements that change the relation between the body and the surrounding spatial configurations of contours, surfaces, events, and objects. This will include manual operations upon objects in the immediate behavioral space, but outside the area of focal vision, that are made during fixation and intense foveal work.

The properties of parietal LS neurons appear suitable to serve several aspects of vision and visual behavior that fall under the rubric of ambient vision. The properties are the large and frequently bilateral response areas of the LS neurons, many of which exclude the foveal region; the striking sensitivity of LS neurons to both stationary and moving stimuli, particularly to the direction of motion; and the opponent organization of the movement vectors. The aspects of visual behavior relate to the central representation of apparent motion of the environment during locomotion and during head movements and to the movement of objects in the periphery of the behavioral space when the eyes are fixed. Both are thought important for the continual updating of a continuing image of the immediately surrounding spatial frame. Finally, we consider the properties of the parietal LS cells in relation to directed visual attention.

Concerning apparent motion, forward locomotion at rates from walking to sprinting (2 to $10 \mathrm{~m} / \mathrm{sec})$ will produce angular velocities of apparent motion of objects, passing within 1 to $2 \mathrm{~m}$, that range from very low values when distant $10 \mathrm{~m}$ or more to several hundred degrees per second as they pass by and out of the visual fields through their peripheral edges. Parietal LS neurons are sensitive to movement over just that range of velocities, from 10 to $800^{\circ} / \mathrm{sec}$. Thus, forward locomotion will activate in an optimal manner the movement-sensitive pa- rietal LS neurons. The flow of the environment by the head will be most heavily represented in the contralateral parietal lobe, but the presence of a significant number of LS neurons with bilateral response areas and opponent vector orientation ensures that the apparent motion of the environment is represented to some degree in both hemispheres. We are uncertain whether this system can provide discriminable signals of the velocity of movement of objects, for the cells that we have studied possessed flat and broad velocity/impulse frequency functions. It may be that the optimal stimuli for these cells are accelerating visual images which we have not used in this study. It is the relative insensitivity to different velocities of the parietal LS cells that leads us to question whether this system plays a significant role in motion perception or in the discrimination between different velocities of motion when these are considered as visual perceptive functions. Whether defects of this discriminative capacity appear in primates after parietal lobe lesions is uncertain and, if present, may be difficult to differentiate from the pervasive visual inattention.

It is obvious that the apparent inward and outward motions of the environment induced by reciprocal movements of the head will activate alternatively the inward and outward sets of directionally sensitive parietal LS neurons. We suggest that these signals of apparent motion of the environment during head movements and during forward locomotion play a role in spatial orientation and perception, considered in the context of ambient vision, and may provide signals used in preserving a perceptual constancy of the environment during movement and in updating a putative neural construct of the surrounding behavioral space and of objects within it. No direct evidence exists for such a neural construct; nevertheless, it is some such neural apparatus that appears to be severely impaired by parietal lobe lesions.

The cardinal signs of the parietal lobe syndrome in primates are contralateral inattention and neglect. The inattention obtains for all major sensory systems and strikingly so for vision. The neglect includes both that to sensory stimuli and a poverty of and errors in motor initiatives into the contralateral behavioral space. That neglect is, however, more a defect in spatial awareness and perception than it is a specific sensory or motor defect. Visual inattention and spatial neglect may occur with intact visual fields and normal visual acuity. We consider them to reflect the loss of the fixation and oculomotor neurons of the parietal lobe and the resulting deficiency in the function of the distributed neural system of which they are parts (Mountcastle, 1976; 1978). These sets of neurons are thought to be involved in the direction of gaze and its accompanying attention to objects of interest. The parietal LS neurons have properties eminently suitable for the afferent elements of such a neural mechanism, particularly because of the large number sensitive to movement of objects inward across the peripheral edges of the visual fields toward the center of gaze. Such a stimulus obviously has a powerful capacity for attracting attention. In addition, the neural activity set in motion by it could have an important survival value. This is so because the attentive primate, sitting or standing and concentrating intently on foveal work, 
would be vulnerable to threatening events unless he possessed a system for detecting signs of them in the far periphery of the visual fields where focal vision, weak in any case, is thought to be suppressed during foveal work. The afferent system leading to the parietal light-sensitive neurons, by contrast, retains its excitability during interested fixation. Indeed, as we shall show in a future paper, that excitability is increased for many neurons during interested fixation. Such a system seems ideally suited to provide signals evoking a shift of attention and gaze from one object to another.

Studies of mammals other than primates have led a number of investigators to suggest that the two components of the visual system are to a certain degree independent and parallel (see, e.g., Schneider, 1969; Sprague et al., 1979). This duality and independence was suggested by Trevarthen (1968) to have its functional parallel in those aspects of visual function that he called focal and ambient. Such a separation of neural mechanisms seems less likely in the primate than it may in other mammals for two reasons. Firstly, there is a greater corticalization of the collicular system in the primate, with a clear cortical projection from the colliculus and the pretectal nuclei, via the dorsal thalamus. Secondly, especially concerning the parietal lobe as a projection target and thus the aspect of visual function in which it is thought to play a part, there is convergence between striate and collicular projections.

However, convergence between systems cannot automatically be taken to mean convergence between elements or between modular groups of elements. This bears on the body of evidence developed in recent years that supports the idea of parallel processing in the visual system (for review, see Stone et al., 1979). This is envisaged to begin with the three classes of ganglion cells labeled $\mathrm{Y}, \mathrm{X}$, and $\mathrm{W}$ that project separately upon different neural elements of the lateral geniculate nucleus and the striate cortex and, indeed, transcortically through this area to certain of its efferent pathways. This model of the visual system does not exclude either a certain degree of convergent interaction among $\mathrm{Y}, \mathrm{X}$, and $\mathrm{W}$ elements nor a certain degree of hierarchical organization, but it does emphasize separate projections and parallel processing. The superior colliculus is thought to receive retinal input mainly from $\mathrm{Y}$ and $\mathrm{W}$ ganglion cells and only sparsely from $\mathrm{X}$ ganglion cells. The functional properties of the $\mathrm{Y}$ and $\mathrm{W}$ cells, together with other evidence obtained in studies of mammals with collicular lesions, led Stone et al. (1979), like Trevarthen (1968), to suggest a parallel functional dependence of focal and ambient vision upon the striate and collicular components of the visual system, respectively.

It has not yet been possible to examine the functional properties of the parietal LS neurons in sufficient detail in waking monkeys to submit them to the criteria of either the Y-X-W or the simple-complex-hypercomplex classification schemes. The extraordinary movement sensitivity, predominantly transient pattern of response, and large response areas recall some of the properties of $\mathrm{Y}$ neurons, but no more certain statement can be made at this time. Thus, we leave open the question to what degree the participation of the parietal lobe in certain aspects of visual function depends upon one or another of the major components of the visual system, or indeed upon both, as the marked convergence between its collicular and striate inputs suggests.

\section{Summary and Conclusions}

We describe in this paper the results of a new study of the inferior parietal lobule in waking monkeys, in which the methods of behavioral control and visual stimulation have been combined with that of single neuron analysis. In the course of this research, 204 successful microelectrode penetrations were made into area 7-a $(\mathrm{PG})$ of the inferior parietal lobule. More than 1600 neurons were identified by isolation of the electrical signs of their action potentials, and more than 800 were studied further in a programmatic manner. A new classification strategy was adopted in which every neuron that responded in any way or under any circumstances to visual stimuli was termed a light-sensitive (LS) neuron, regardless of other properties. Of the cells brought under study, $31 \%$ (529) were thus classified as LS neurons; of these, a small proportion $(67 / 529)$ displayed other properties as well; under our earlier classification schemes, they would have been called "complex" cells. Neurons found insensitive to visual or to any other form of passive stimulation fell into classes defined in our previous studies of the parietal lobe: the fixation neurons (17\%), the oculomotor neurons $(14 \%)$, and the projection-manipulation neurons $(12 \%)$. We emphasize that for each of these latter classes, one of the defining characteristics is the absence of response to visual stimuli. Of the neurons that we brought under study, $31 \%$ escaped identification even though they were examined intensely, all by "clinical" examination and many in programmed behavioral runs with tests for oculomotor and visual correlations.

The light-sensitive cells of area 7 were studied under low photopic to mesopic conditions, with stimuli usually about $1 \mathrm{log}$ unit above background. They fell into two classes in regard to the spatial distribution of their response areas, when tested with relatively large stimuli $\left(6^{\circ} \times 6^{\circ}\right)$. The largest group $(59 \%)$ was activated from very large areas that were frequently bilateral and which exhibited the property of foveal sparing; i.e., stimuli centered over the point of fixation evoked no responses. The remainder were related to response areas which we term foveal-inclusive; for them, $6^{\circ} \times 6^{\circ}$ stimuli centered on the fixation point did evoke responses, and for some, the intensity of response was maximal for foveally centered lights and decreased symmetrically on either side. However, only eight of 216 cells that we examined in detail showed any response to the fixation target light itself. This suggests that the large majority of cells that we have classed as foveal-inclusive on the basis of studies with large stimuli may also have small central zones of inexcitability, like neurons with foveal sparing.

The most striking characteristic of parietal LS neurons is their sensitivity to the movement of visual stimuli, particularly to the direction of movement. The velocity sensitivity range varies from 10 to $800^{\circ} / \mathrm{sec}$, but parietal neurons provide only a poorly discriminable signal of differences in velocity. The directional vectors of parietal neurons are arranged in a systematic way, pointing either 
inward toward the center of the visual field or outward toward the perimeter. For neurons that subtend bilateral response areas, the directional vectors in the two halffields most commonly point in opposite directions and are frequently arranged in a radial manner. We term this arrangement opponent vector organization.

The results obtained are discussed in relation to defects in visual and visuomotor behavior that occur in primates with parietal lobe lesions, particularly those of hemiinattention and neglect. The light-sensitive neurons of area 7 possess properties well suited for signaling motion in the immediate behavioral surround and of the apparent motion that accompanies head movements and forward locomotion. Thus, they are surmised to contribute to a continual updating of a central neural image of the spatial frame of the immediate behavioral surround and to the perceptual constancy of that space that obtains during body movement. The light-sensitive neurons possess properties suitable for the attraction of gaze and attention toward objects and events in peripheral visual fields.

It is this system together with the sets of parietal neurons concerned with initiatives toward action, the fixation, oculomotor, and projection-manipulation neurons, whose destruction is thought to account, at least in part, for the cardinal features of the parietal lobe syndrome.

\section{References}

Andersen, R. A., and V. B. Mountcastle (1980) The direction of gaze influences the response of light sensitive neurons of the inferior parietal lobule (area 7) in waking monkeys. Soc. Neurosci. Abstr. 6: 673.

Baleydier, D., and F. Mauguiere (1977) Pulvinar-latero posterior afferents to cortical area 7 in monkeys demonstrated by horseradish peroxidase tracing technique. Exp. Brain Res. 27: 501-507.

Benevento, L. A., and B. Davis (1977) Topographical projections of the prestriate cortex to the pulvinar nuclei in the macaque monkey: An autoradiographic study. Exp. Brain Res. 30: 405-424.

Benevento, L. A., and J. H. Fallon (1975) The ascending projections of the superior colliculus in the rhesus monkey (Macaca mulatta). J. Comp. Neurol. 160: 339-362.

Benevento, L. A., and M. Rezak (1976) The cortical projections of the inferior pulvinar and adjacent lateral pulvinar in the rhesus monkey (Macaca mulatta): An autoradiographic study. Brain Res. 108: 1-24.

Benevento, L. A., and M. Rezak (1977) Further observations on the projections of the layers of the superior colliculus in the rhesus monkey with autoradiographic tracing methods. Soc. Neurosci. Abstr. 3: 553.

Benevento, L. A., M. Rezak, and R. Santos-Anderson (1977) An autoradiographic study of the projections of the pretectum in the rhesus monkey (Macaca mulatta): Evidence for sensorimotor links to the thalamus and oculomotor nuclei. Brain Res. 127: 197-218.

Cynader, M., and N. Berman (1972) Receptive-field organization of monkey superior colliculus. J. Neurophysiol. 35: 187201.

Desimone, R., and C. G. Gross (1979) Visual areas in the temporal cortex of the macaque monkey. Brain Res. 178: $363-380$.

Desimone, R., C. Bruce, and C. G. Gross (1979) Neurons in the superior temporal sulcus of the macaque still respond to visual stimuli after removal of striate cortex. Soc. Neurosci. Abstr. 5: 781.

Divac, I., J. H. LaVail, P. Rakic, and K. R. Winston (1977) Heterogeneous afferents to the inferior parietal lobule of the rhesus monkey revealed by the retrograde transport method. Brain Res. 123: 197-207.

Dubner, R., and S. M. Zeki (1971) Response properties and receptive fields of cells in an anatomically defined region of the superior temporal sulcus in the monkey. Brain Res. 35: 528-532.

Goldberg, M. E., and D. L. Robinson (1977) Visual responses of neurons in monkey inferior parietal lobule: The physiologic substrate of attention and neglect. Neurology (NY) 27: 350.

Goldberg, M. E., and D. L. Robinson (1978) Visual systems: Superior colliculus. In Handbook of Behavioral Neurology. Vol. 1: Sensory Integration, R. B. Masterton, ed., pp. 119164, Plenum Press, New York.

Goldberg, M. E., and R. H. Wurtz (1972) Activity of superior colliculus in behaving monkey. I. Visual receptive fields of single neurons. J. Neurophysiol. 35: 542-559.

Gross, C. G., D. B. Bender, and C. E. Rocha-Miranda (1969) Visual receptive fields of neurons in inferotemporal cortex of the monkey. Science 166: 1303-1306.

Hubel, D. H., and 'T. N. Wiesel (1968) Receptive fields and functional architecture of monkey striate cortex. J. Physiol. (Lond.) 195: 215-243.

Hubel, D. H., and T. N. Wiesel (1974) Uniformity of monkey striate cortex: A parallel relationship between field size, scatter, and magnification factor. J. Comp Neurol. 158: 295-306.

Hyvarinen, J., and A. Poranen (1974) Function of the parietal associative area 7 as revealed from cellular discharges in alert monkeys. Brain 97: 673-692.

Hyvarinen, J., and Y. Shelepin (1979) Distribution of visual and somatic functions in the parietal associative area 7 of the monkey. Brain Res. 169: 561-564.

Jones, E. G., and T. P. S. Powell (1970) An anatomical study of converging sensory pathways within the cerebral cortex of the monkey. Brain 93: 793-820.

Kasdon, D. L., and S. Jacobson (1978) The thalamic afferents to the inferior parietal lobule of the rhesus monkey. J. Comp. Neurol. 177: 685-706.

Kawano, K., M. Sasaki, and M. Yamashita (1980) Vestibular input to visual tracking neurons in the posterior parietal association cortex of the monkey. Neurosci. Lett. 17: 55-60.

Leinonen, L., and G. Nyman (1979) II. Functional properties of cells in anterolateral part of area 7 associative face area of awake monkeys. Exp. Brain Res. 34: 321-333.

Leinonen, L., J. Hyvarinen, G. Nyman, and I. Linnankoski (1979) I. Functional properties of neurons in lateral part of associative area 7 in awake monkeys. Exp. Brain Res. 34: 299-320.

Lynch, J. C. (1980) The functional organization of the posterior parietal association cortex. Behav. Brain Sci., in press.

Lynch, J. C., C. Acuna, H. Sakata, A. Georgopoulos, and V. B. Mountcastle (1973a) The parietal association area and immediate extrapersonal space. Soc. Neurosci. Abstr. 3: 244.

Lynch, J. C., H. Sakata, A. Georgopoulos, and V. B. Mountcastle (1973b) Parietal association cortex neurons active during hand and eye tracking of objects in immediate extrapersonal space. Physiologist 16: 384 .

Lynch, J. C., V. B. Mountcastle, W. H. Talbot, and T. C. T. Yin (1977) Parietal lobe mechanısms for directed visual attention. J. Neurophysiol. 40: 362-389.

Magnuson, D. J., M. Rezak, and L. A. Benevento (1979) Some observations on the organization of the retinal projections to the pretectum and superior colliculus in the macaque monkey as demonstrated by the combined use of laser beam lesions of the retina and autoradiography. Soc. Neurosci. Abstr. 5: 
794.

Marrocco, R. T., and R. H. Li (1977) Monkey superior colliculus: Properties of single cells and their afferent inputs. J. Neurophysiol. 40: 844-860.

Mesulam, M. M., G. W. Van Hoesen, D. N. Pandya, and N. Geschwind (1977) Limbic and sensory connections of the inferior parietal lobule (area PG) in the rhesus monkey: A study with a new method for horseradish peroxidase histochemistry. Brain Res. 136: 393-414.

Montero, V. M. (1980) Patterns of connections from the striate cortex to cortical visual areas in superior temporal sulcus of macaque and middle temporal gyrus of owl monkey. J. Comp. Neurol. 189: 45-59.

Motter, B. C., and V. B. Mountcastle (1979) Afferent visual signals for directed visual attention. Soc. Neurosci. Abstr. 5 : 118.

Motter, B. C., and V. B. Mountcastle (1980) Active directed gaze controls the excitability of the light sensitive neurons of the inferior parietal lobule in the waking monkey. Soc. Neurosci. Abstr. 6: 673.

Mountcastle, V. B. (1976) The world around us: Neural command functions for selective attention. Neurosci. Res. Program Bull. 14: 1-47.

Mountcastle, V. B. (1978) Brain mechanisms for directed attention. J. R. Soc. Med. 71: 14-28.

Mountcastle, V. B., J. C. Lynch, A. Georgopoulos, H. Sakata, and C. Acuna (1975) Posterior parietal association cortex of the monkey: Command functions for operations within extrapersonal space. J. Neurophysiol. 38: 871-908.

Pandya, D. N., and H. G. J. M. Kuypers (1969) Cortico-cortical connections in the rhesus monkey. Brain Res. 13: 13-36.

Pandya, D. N., and L. A. Vignolo (1969) Interhemispheric projections of the parietal lobe in the rhesus monkey. Brain Res. 15: 49-65.

Partlow, G. D., M. Colonnier, and J. Szaba (1977) Thalamic projections of the superior colliculus in the rhesus monkey, Macaca mulatta. A light and electron microscopic study. J. Comp. Neurol. 171: 285-318.

Pearson, R. C. A., P. Brodal, and T. P. S. Powell (1978) The projection of the thalamus upon the parietal lobe in the monkey. Brain Res. 144: 143-148.

Petras, J. M. (1971) Connections of the parietal lobe. J. Psychiatr. Res. 8: 189-201.

Poggio, G. F., and W. H. Talbot (1980) Mechanisms of static and dynamic stereopsis in foveal cortex of the rhesus monkey. J. Physiol. (Lond.), in press.

Poggio, G. F., F. H. Baker, R. J. W. Mansfield, A. Sillito, and P. Grigg (1975) Spatial and chromatic properties of neurons subserving foveal and parafoveal vision in rhesus monkey. Brain Res. 100: 25-59.

Posner, M. I., M. J. Nissen, and W. C. Ogden, (1978) Attended and unattended processing modes: The role of set for spatial location. In Modes of Perceiving and Processing Information, H. L. Pick and I. J. Saltzman, eds., pp. 137-157, Lawrence Erlbaum Associates, Hillsdale, NJ.

Rezak, M., and L. A. Benevento (1975) Cortical projections of corticorecipient and tectorecipient zones of the pulvinar in the macaque monkey. Soc. Neurosci. Abstr. 1: 63.

Rezak, M., and L. A. Renevento (1977) A redefinition of pulvinar subdivisions in the macaque monkey: Evidence for three distinct subregions within classically defined lateral pulvinar. Soc. Neurosci. Abstr. 3: 574.

Robinson, D. L., and M. E. Goldberg (1977a) Functional properties of posterior parietal cortex of the monkey. I. Sensory responses. Soc. Neurosci. Abstr. 3:574

Robinson, D. L., and M. E. Godberg (1977b) Visual properties of neurons in the parietal cortex of the awake monkey. Invest. Opthalmol. Vis. Sci. 16 (Suppl.): 156.
Robinson, D. L., M. E. Goldberg, and G. B. Stanton (1978) Parietal association cortex in the primate: Sensory mechanisms and behavioral modulations. J. Neurophysiol. 41: 910932.

Rocha-Miranda, C. E., D. B. Bender, C. G. Gross, and M. Mishkin (1975) Visual activation of neurons in inferotemporal cortex depends on striate cortex and forebrain commissures. J. Neurophysiol. 38: 475-491.

Rolls, E. T., D. Perrett, S. J. Thorpe, A. Puerto, A. Roper-Hall, and S. Maddison (1979) Responses of neurons in area 7 of the parietal cortex to objects of different significance. Brain Res. 169: 194-198.

Sakata, H., H. Sibutani, and K. Kawano (1977) Spatial selectivities of "visual" neurons in the posterior parietal association cortex of the monkey. Proc. IUPS XIII 652.

Sakata, H., H. Shibutani, and K. Kawano (1978) Parietal neurons with dual sensitivity to real and induced movements of visual target. Neurosci. Lett. 9: 165-169.

Sakata, H., H. Shibutani, and K. Kawano (1980) Spatial properties of visual fixation neurons in posterior parietal association cortex of the monkey. J. Neurophysiol. 43: 1654-1672.

Schiller, P. H., and F. Koerner (1971) Discharge characteristics of single units in the superior colliculus of the alert rhesus monkey. J. Neurophysiol. 34: 920-936.

Schiller, P. H., M. Stryker, M. Cynader, and N. Berman (1974) Response characteristics of single cells in the monkey superior colliculus following ablation or cooling of visual cortex. J. Neurophysiol. 37: 181-194.

Schiller, P. H., B. L. Finlay, and S. P. Volman (1976a) Quantitative studies of single-cell properties in monkey striate cortex. I. Spatiotemporal organization of receptive fields. J. Neurophysiol. 39: 1288-1319.

Schiller, P. H., B. L. Finlay, and S. F. Volman (1976b) Quantitative studies of single-cell properties in monkey striate cortex. II. Orientation specificity and ocular dominance. J. Neurophysiol. 39: 1320-1333.

Schneider, G. E. (1969) Two visual systems. Science 163: 895902.

Seltzer, B., and D. Pandya (1978) Afferent cortical connections and architectonics of the superior temporal sulcus and surrounding cortex in the rhesus monkey. Brain Res. 149:1-24.

Seltzer, B., and D. N. Pandya (1980) Converging visual and somatic sensory cortical input to the intraparietal sulcus of the rhesus monkey. Brain Res. 192: 339-351.

Sprague, J. M., M. A. Berkley, and H. C. Hughes (1979) Visual acuity functions and pattern discrimination in the destriate cat. Acta Neurobiol. Exp. 39: 643-682.

Stanton, G. V., W. L. R. Cruce, M. E. Goldberg, and D. L. Robinson (1977) Some ipsilateral projections to areas PF and PG of the inferior parietal lobule in monkeys. Neurosci. Lett. 6: 243-250.

Stone, J., B. Dreher, and A. Leventhal (1979) Hierarchical and parallel mechanisms in the organization of visual cortex. Brain Res. Rev. 1: 345-394.

Trevarthen, C. B. (1968) Two mechanisms of vision in primates. Psychol. Forsch. 31: 299-337.

'Irojanowski, J. Q., and S. Jacobson (1976) Areal and laminar distribution of some pulvinar cortical efferents in the rhesus monkey. J. Comp. Neurol. 169: 371-392.

Ungerleider, L. G., and M. Mishkin (1978) Interactions of striate and posterior parietal cortex in spatial vision. Soc. Neurosci. Abstr. 4: 649.

Ungerleider, L. G., and M. Mishkin (1979) The striate projection zone in the superior temporal sulcus of Macaca mulatta: Location and topographic organization. J. Comp. Neurol. 188: 347-366.

Updyke, B. V. (1974) Characteristics of unit responses in superior colliculus of the Cebus monkey. J. Neurophysiol. 37: 
896-909.

von Bonin, G., and P. Bailey (1947) The Neocortex of Macaca mulatta. University of Illinois Press, Urbana.

Weller, R. E., and J. H. Kaas (1978) Connections of striate cortex with the posterior bank of the superior temporal sulcus in macaque monkeys. Soc. Neurosci. Abstr. 4: 650.

Yin, T. C. T., and V. B. Mountcastle (1977) Visual input to the visuomotor mechanisms of the monkey's parietal lobe. Science 197: 1381-1383.
Zeki, S. M. (1974) Functional organization of a visual area in the posterior bank of the superior temporal sulcus of the rhesus monkey. J. Physiol. (Lond.) 236: 549-573.

Zeki, S. M. (1975) The functional organization of projections from striate to prestriate visual cortex in the rhesus monkey. Cold Spring Harbor Symp. Quant. Biol. 40: 591-600.

Zeki, S. M. (1976) The projections to the superior temporal sulcus from areas 17 and 18 in the rhesus monkey. Proc. $R$. Soc. Lond. (Biol.) 193: 199-207. 